\title{
ON WEAK COMPLETENESS OF THE SET OF ENTROPY SOLUTIONS TO A DEGENERATE NON-LINEAR PARABOLIC EQUATION*
}

\author{
E. YU. PANOV ${ }^{\dagger}$
}

\begin{abstract}
We prove that weak limits of approximate entropy solutions to a one-dimensional degenerate parabolic equation are entropy solutions as well.
\end{abstract}

1. Introduction. In the strip $\Pi_{T}=(0, T) \times \mathbb{R}, T>0$, we consider the nonlinear parabolic equation

$$
u_{t}+\varphi(u)_{x}-g(u)_{x x}=0
$$

where the functions $\varphi(u), g(u) \in C(\mathbb{R})$, and $g(u)$ is non-strictly increasing. Since $g(u)$ may be constant on non-degenerate intervals, (1.1) is a degenerate parabolic equation. In particular, for $g(u) \equiv$ const this equation reduces to the first-order conservation law

$$
u_{t}+\varphi(u)_{x}=0
$$

We recall the notions of weak and entropy solutions of $(1.1)$ (see $[2,10,1])$.

Definition 1.1. A bounded measurable function $u=u(t, x) \in L^{\infty}\left(\Pi_{T}\right)$ is called a weak solution of (1.1) if the generalized derivative $g(u)_{x} \in L_{l o c}^{2}\left(\Pi_{T}\right)$ and

$$
u_{t}+\varphi(u)_{x}-g(u)_{x x}=0
$$

in the sense of distributions on $\Pi_{T}\left(\right.$ in $\left.\mathcal{D}^{\prime}\left(\Pi_{T}\right)\right)$. A weak solution $u=u(t, x)$ of (1.1) is called an entropy solution of this equation if for each $k \in \mathbb{R}$

$$
|u-k|_{t}+[\operatorname{sign}(u-k)(\varphi(u)-\varphi(k))]_{x}-|g(u)-g(k)|_{x x} \leq 0 \quad \text { in } \mathcal{D}^{\prime}\left(\Pi_{T}\right) .
$$

Condition (1.4) means that for any non-negative test function $f=f(t, x) \in C_{0}^{\infty}\left(\Pi_{T}\right)$

$$
\int_{\Pi_{T}}\left\{|u-k| f_{t}+[\operatorname{sign}(u-k)(\varphi(u)-\varphi(k))] f_{x}+|g(u)-g(k)| f_{x x}\right\} d t d x \geq 0 .
$$

In the case of equation (1.2) condition (1.4) coincides with the known Kruzhkov entropy condition [9]. If the function $g(u)$ strictly increases then any weak solution of (1.1) is an entropy solution of this equation as well (cf. [2]) but for degenerate equations this may be violated and, in particular, entropy condition (1.4) is necessary for the uniqueness of solution to the Cauchy problem for equation (1.1).

Remark also that relation (1.3) readily follows from entropy condition (1.4) with $k= \pm M$, where $M \geq\|u\|_{\infty}$.

Now we consider a bounded in $L^{\infty}\left(\Pi_{T}\right)$ sequence $u_{n}=u_{n}(t, x)$ of entropy solutions of (1.1) weakly convergent to $u=u(t, x) \in L^{\infty}\left(\Pi_{T}\right)$. In the case of a conservation law (1.2) with the flux $\varphi(u) \in C^{1}(\mathbb{R})$ it is rather well known that $u=u(t, x)$ is a weak

${ }^{*}$ This research was carried out with the financial support of the Russian Foundation for Basic Research (grant 09-01-00490).

${ }^{\dagger}$ Novgorod State University, B. Sankt-Peterburgskaya, 41, 173003 Veliky Novgorod, Russia (Eugeny.Panov@novsu.ru). 
solution of (1.2). This is a simple application of the Tartar-Murat compensated compactness theory (for the proof we refer to $[4,19]$; see also books $[5,18]$ ). The problem whether this solution is an entropy solution was positively solved only recently, in [15]. Now, we extend this result to the case of degenerated parabolic equations (1.1).

More generally, we suppose that $u_{n}$ is an entropy solution of the approximate equation

$$
u_{t}+\varphi_{n}(u)_{x}-g_{n}(u)_{x x}=0,
$$

where the sequences $\varphi_{n}(u), g_{n}(u) \in C(\mathbb{R}), n \in \mathbb{N}$, converge as $n \rightarrow \infty$ to $\varphi(u), g(u)$, respectively, uniformly on any segment in $\mathbb{R}$. Naturally, it is assumed that the functions $g_{n}(u)$ are (non-strictly) increasing for all $n \in \mathbb{N}$. Supposing that $u_{n} \rightarrow u$ as $n \rightarrow \infty$ weakly-* in $L^{\infty}\left(\Pi_{T}\right)$, we are going to prove that the limit function $u=u(t, x)$ is an entropy solution of equation (1.1). Moreover, this entropy solution is actually the entropy solution of the Cauchy problem for equation (1.1) with some initial data $u_{0}=u_{0}(x) \in L^{\infty}(\mathbb{R})$ understood in the sense of relation

$$
\underset{t \rightarrow 0}{\operatorname{ess}} \lim u(t, \cdot)=u_{0} \text { in } L_{l o c}^{1}(\mathbb{R}) .
$$

To prove this result, we use, as in [15], the compensated compactness method. But now we need the new version of this method adapted to the case of inhomogeneous differential constraints and developed in recent paper [17].

2. Preliminaries. In the sequel, we will need the following two lemmas.

LEMMA 2.1. Let $u=u(t, x)$ be an entropy solution of $(1.1), M=\|u\|_{\infty}$. Then for each $k \in \mathbb{R}$

$$
|u-k|_{t}+[\operatorname{sign}(u-k)(\varphi(u)-\varphi(k))]_{x}-|g(u)-g(k)|_{x x}=-\mu_{k} \quad \text { in } \mathcal{D}^{\prime}\left(\Pi_{T}\right),
$$

where $\mu_{k}$ is a non-negative locally finite Borel measure on $\Pi_{T}$. Besides, for every compact $K \subset \Pi_{T}$

$$
\mu_{k}(K) \leq C(K)\left(M+\max _{|u| \leq M}|\varphi(u)|+\max _{|u| \leq M}|g(u)|\right),
$$

where $C(K)$ is a positive constant depending only on $K$.

Proof. Relation (2.1) follows from the known representation of non-negative distributions. Let $K \subset \Pi_{T}$ be a compact set. If $|k|>M$ then

$$
\mu_{k}=-\left(u_{t}+\varphi_{n}(u)_{x}-g_{n}(u)_{x x}\right)=0
$$

and (2.2) holds for each positive constant $C(K)$. Assuming that $|k| \leq M$, we chose a test function $f=f_{K} \in C_{0}^{\infty}\left(\Pi_{T}\right)$ such that $f \geq 0$ and $f \equiv 1$ on $K$. Then it follows from (2.1) that

$$
\begin{array}{r}
\mu^{k}(K) \leq \int_{\Pi_{T}} f d \mu_{k}(t, x)= \\
\int_{\Pi_{T}}\left\{|u-k| f_{t}+[\operatorname{sign}(u-k)(\varphi(u)-\varphi(k))] f_{x}+|g(u)-g(k)| f_{x x}\right\} d t d x \leq \\
2 \int_{\Pi_{T}} \max \left\{\left|f_{t}\right|,\left|f_{x}\right|,\left|f_{x x}\right|\right\} d t d x\left(M+\max _{|u| \leq M}|\varphi(u)|+\max _{|u| \leq M}|g(u)|\right)= \\
C(K)\left(M+\max _{|u| \leq M}|\varphi(u)|+\max _{|u| \leq M}|g(u)|\right),
\end{array}
$$


with

$$
C(K)=2 \int_{\Pi_{T}} \max \left\{\left|f_{t}\right|,\left|f_{x}\right|,\left|f_{x x}\right|\right\} d t d x, f=f_{K} .
$$

The proof is complete.

Lemma 2.2. Let $u=u(t, x)$ be a weak solution of (1.1), $M=\|u\|_{\infty}, a, b \in$ $[-M, M], a<b$. Then for any $f=f(t, x) \in C_{0}^{\infty}\left(\Pi_{T}\right), f \geq 0$

$\int_{a \leq u(t, x) \leq b}\left(g(u)_{x}\right)^{2} f d t d x \leq C(f)\left(2 M+3 \max _{|u| \leq M}|\varphi(u)|+2 \max _{|u| \leq M}|g(u)|\right) \max _{a \leq u \leq b}|g(u)|$,

where $C(f)$ is a constant depending only on $f$.

Proof. Let $s_{a, b}(u)=\max (a, \min (b, u))$ be the cut-off function, and $G(u)=$ $\int_{a}^{u} g\left(s_{a, b}(v)\right) d v$. Observe that $g\left(s_{a, b}(u)\right)=\max (g(a), \min (g(b), g(u)))=s(g(u))$, where $s(v) \doteq s_{g(a), g(b)}(v)$. By the chain rule for Sobolev functions (see, for instance, [7, Chapter 4])

$$
s(g(u))_{x}=\tilde{\chi}(g(u)) g(u)_{x}=\chi(u) g(u)_{x} \in L_{l o c}^{2}\left(\Pi_{T}\right)
$$

where $\tilde{\chi}(v), \chi(u)$ are the indicator functions of the segments $[g(a), g(b)],[a, b]$, respectively. Here we also take into account that $g(u)_{x}=0$ almost everywhere on the sets $g^{-1}(a) \cup g^{-1}(b)$ (cf. [7, Chapter 4]).

By $\left[2\right.$, Lemma 4] we claim that for each $f=f(t, x) \in C_{0}^{\infty}\left(\Pi_{T}\right)$

$$
\begin{array}{r}
\int_{\Pi_{T}} G(u) f_{t}=-\left\langle u_{t}, s(g(u)) f\right\rangle=\left\langle\varphi(u)_{x}-g(u)_{x x}, s(g(u)) f\right\rangle= \\
-\int_{\Pi_{T}}\left(\varphi(u)-g(u)_{x}\right)(s(g(u)) f)_{x} d t d x= \\
-\int_{\Pi_{T}}\left\{\varphi(u) \chi(u) g(u)_{x} f+\varphi(u) s(g(u)) f_{x}-\right. \\
\left.\chi(u)\left(g(u)_{x}\right)^{2} f-g(u)_{x} s(g(u)) f_{x}\right\} d t d x
\end{array}
$$

We introduce the function

$$
H(u)=\int_{a}^{s_{a, b}(u)} \varphi(s) d g(s)=\int_{g(a)}^{s(g(u))} \varphi\left(g_{0}^{-1}(v)\right) d v
$$

where $g_{0}^{-1}(v)$ is an element in the set $g^{-1}(v)$ of minimal absolute value. Remark that by the chain rule

$$
H(u)_{x}=\varphi\left(g_{0}^{-1}(g(u))\right) \chi(u) g(u)_{x} \in L_{l o c}^{2}\left(\Pi_{T}\right) .
$$

Since the set $E$ of discontinuity points of the increasing function $g_{0}^{-1}(v)$ is at most countable then $g(u)_{x}=0$ almost everywhere on the set $g^{-1}(E)$. On the other hand, $g_{0}^{-1}(g(u))=u$ if $g(u) \notin E$. Hence, $H(u)_{x}=\varphi(u) \chi(u) g(u)_{x}$ and, integrating by part, we arrive at

$$
\int_{\Pi_{T}} \varphi(u) \chi(u) g(u)_{x} f d t d x=\int_{\Pi_{T}} H(u)_{x} f d t d x=-\int_{\Pi_{T}} H(u) f_{x} d t d x .
$$


Similarly, denoting $p(v)=\int_{g(a)}^{v} s(v) d v$, we get the relation

$$
\int_{\Pi_{T}} g(u)_{x} s(g(u)) f_{x} d t d x=\int_{\Pi_{T}} p(g(u))_{x} f_{x} d t d x=-\int_{\Pi_{T}} p(g(u)) f_{x x} d t d x .
$$

Using the above relations, we derive from (2.4) that

$$
\int_{\Pi_{T}} \chi(u)\left(g(u)_{x}\right)^{2} f d t d x=\int_{\Pi_{T}}\left\{G(u) f_{t}+\psi(u) f_{x}+p(g(u)) f_{x x}\right\} d t d x,
$$

where we denote

$$
\psi(u)=\varphi(u) s(g(u))-H(u)=\varphi(u) s(g(u))-\int_{g(a)}^{s(g(u))} \varphi\left(g_{0}^{-1}(v)\right) d v .
$$

Equality (2.5) implies that for any $f=f(t, x) \in C_{0}^{\infty}\left(\Pi_{T}\right), f \geq 0$

$$
\begin{array}{r}
\int_{\Pi_{T}} \chi(u)\left(g(u)_{x}\right)^{2} f d t d x \leq \\
\max _{|u| \leq M}(|G(u)|+|\psi(u)|+\mid p(g(u) \mid)) \int_{\Pi_{T}} \max \left\{\left|f_{t}\right|,\left|f_{x}\right|,\left|f_{x x}\right|\right\} d t d x .
\end{array}
$$

Remark that

$$
\begin{aligned}
& |G(u)| \leq 2 M \max _{a \leq u \leq b}|g(u)|, \quad|\psi(u)| \leq 3 \max _{|u| \leq M}|\varphi(u)| \max _{a \leq u \leq b}|g(u)|, \\
& |p(g(u))| \leq|g(u)-g(a)| \max |s(v)| \leq 2 \max _{|u| \leq M}|g(u)| \max _{a \leq u \leq b}|g(u)| .
\end{aligned}
$$

It follows from these estimates and (2.6) that

$$
\int_{\Pi_{T}} \chi(u)\left(g(u)_{x}\right)^{2} f d t d x \leq C(f)\left(2 M+3 \max _{|u| \leq M}|\varphi(u)|+2 \max _{|u| \leq M}|g(u)|\right) \max _{a \leq u \leq b}|g(u)|,
$$

where $C(f)=\int_{\Pi_{T}} \max \left\{\left|f_{t}\right|,\left|f_{x}\right|,\left|f_{x x}\right|\right\} d t d x$. The proof is complete.

In addition to the above lemma we observe that $\max _{a \leq u \leq b}|g(u)|=\max \{|g(a)|,|g(b)|\}$, by monotonicity of $g(u)$.

We will need the notion of a measure-valued function. Recall (see $[6,19])$ that a measure-valued function on $\Pi_{T}$ is a weakly measurable map $(t, x) \mapsto \nu_{t, x}$ of $\Pi_{T}$ into the space $\operatorname{Prob}_{0}(\mathbb{R})$ of probability Borel measures with compact support in $\mathbb{R}$.

The weak measurability of $\nu_{t, x}$ means that for each continuous function $g(\lambda)$ the function $(t, x) \rightarrow\left\langle\nu_{t, x}(\lambda), g(\lambda)\right\rangle=\int g(\lambda) d \nu_{t, x}(\lambda)$ is measurable on $\Pi_{T}$.

We say that a measure-valued function $\nu_{t, x}$ is bounded if there exists $R>0$ such that $\operatorname{supp} \nu_{t, x} \subset[-R, R]$ for almost all $(t, x) \in \Pi_{T}$. We shall denote by $\left\|\nu_{t, x}\right\|_{\infty}$ the smallest such $R$.

Finally, we say that measure-valued functions of the kind $\nu_{t, x}(\lambda)=\delta(\lambda-u(t, x))$, where $u(t, x) \in L^{\infty}\left(\Pi_{T}\right)$ and $\delta\left(\lambda-u^{*}\right)$ is the Dirac measure at $u^{*} \in \mathbb{R}$, are regular. We identify these measure-valued functions and the corresponding functions $u(t, x)$, so that there is a natural embedding $L^{\infty}\left(\Pi_{T}\right) \subset \mathrm{MV}\left(\Pi_{T}\right)$, where $\mathrm{MV}\left(\Pi_{T}\right)$ is the set of bounded measure-valued functions on $\Pi_{T}$. 
Measure-valued functions naturally arise as weak limits of bounded sequences in $L^{\infty}\left(\Pi_{T}\right)$ in the sense of the following theorem by Tartar (see [19]).

TheOREM 2.3. Let $u_{m}(t, x) \in L^{\infty}\left(\Pi_{T}\right), m \in \mathbb{N}$, be a bounded sequence. Then there exist a subsequence $u_{n}(t, x)$ and a measure-valued function $\nu_{t, x} \in \mathrm{MV}\left(\Pi_{T}\right)$ such that

$$
\forall p(\lambda) \in C(\mathbb{R}) \quad p\left(u_{n}\right) \underset{n \rightarrow \infty}{\rightarrow}\left\langle\nu_{t, x}(\lambda), p(\lambda)\right\rangle \quad \text { weakly-* in } L^{\infty}\left(\Pi_{T}\right) .
$$

Besides, $\nu_{t, x}$ is regular, i.e., $\nu_{t, x}(\lambda)=\delta(\lambda-u(t, x))$ if and only if $u_{n}(t, x) \underset{n \rightarrow \infty}{\rightarrow} u(t, x)$ in $L_{l o c}^{1}\left(\Pi_{T}\right)$.

3. Applications of compensated compactness. Now, we suppose that $u_{n}=$ $u_{n}(t, x)$ is a bounded in $L^{\infty}\left(\Pi_{T}\right)$ sequence of weak solutions to approximate equations (1.6). Extracting a subsequence, if necessary, we may suppose that the sequence $u_{n}$ weakly converges to a measure-valued function $\nu_{t, x} \in \mathrm{MV}\left(\Pi_{T}\right)$ in the sense of relation (2.7). Let $M=\sup \left\|u_{n}\right\|_{\infty}$. Then $\left\|\nu_{t, x}\right\|_{\infty} \leq M$.

THEOREM 3.1. For almost every $(t, x) \in \Pi_{T}$ the function $g(u)$ is constant on the convex hull $\operatorname{cosupp} \nu_{t, x}$ of the closed support $\operatorname{supp} \nu_{t, x}$.

Proof. Let $v_{n}=\varphi_{n}\left(u_{n}\right), w_{n}=g_{n}\left(u_{n}\right)$. By our assumptions $\varphi_{n}(u) \rightarrow \varphi(u)$, $g_{n}(u) \rightarrow g(u)$ as $n \rightarrow \infty$ uniformly on $[-M, M]$. This readily implies that $v_{n}-$ $\varphi\left(u_{n}\right) \rightarrow 0, w_{n}-g\left(u_{n}\right) \rightarrow 0$ as $n \rightarrow \infty$ strongly in $L^{\infty}\left(\Pi_{T}\right)$. Therefore weak-* limits $v, w$ of the sequences $v_{n}, w_{n}$ coincide with weak- $*$ limits of $\varphi\left(u_{n}\right), g\left(u_{n}\right)$, respectively, and, in view of $(2.7)$,

$$
v=\left\langle\nu_{t, x}(\lambda), \varphi(\lambda)\right\rangle, \quad w=\left\langle\nu_{t, x}(\lambda), g(\lambda)\right\rangle
$$

Since $u_{n}$ is a weak solution to approximate equation (1.6) then

$$
\left(u_{n}\right)_{t}+\left(v_{n}\right)_{x}-\left(w_{n}\right)_{x x}=0 \text { in } \mathcal{D}^{\prime}\left(\Pi_{T}\right) .
$$

Let us introduce the set

$$
\Lambda=\left\{\lambda=\left(\lambda_{1}, \lambda_{2}, \lambda_{3}\right) \in \mathbb{C}^{3} \mid \exists \xi=(\tau, \xi) \in \mathbb{R}^{2} \backslash\{0\} \quad i \lambda_{1} \tau+\xi^{2} \lambda_{3}=0\right\},
$$

here $i=\sqrt{-1}$. As is easy to see, the quadratic functional $q(\lambda)=\operatorname{Re}\left(\lambda_{1} \overline{\lambda_{3}}\right) \equiv 0$ on the set $\Lambda$. By [17, Corollary 3.2] we can conclude that this functional is weakly continuous on the sequence $\left(u_{n}, v_{n}, w_{n}\right)$, that is, $q\left(u_{n}, v_{n}, w_{n}\right) \underset{n \rightarrow \infty}{\rightarrow} q(u, v, w)$ weakly-* in $L^{\infty}\left(\Pi_{T}\right)$. This means that

$$
u_{n} g_{n}\left(u_{n}\right)=u_{n} w_{n} \underset{n \rightarrow \infty}{\rightarrow} u w \text { weakly-* in } L^{\infty}\left(\Pi_{T}\right) .
$$

It is clear that the sequence $u_{n} g_{n}\left(u_{n}\right)$ has the same weak limit as $u_{n} g\left(u_{n}\right)$. By $(2.7)$ the latter is $\left\langle\nu_{t, x}(\lambda), \lambda g(\lambda)\right\rangle$. Then it follows from (3.2) that

$$
\left\langle\nu_{t, x}(\lambda), \lambda g(\lambda)\right\rangle=u w=\left\langle\nu_{t, x}(\lambda), \lambda\right\rangle\left\langle\nu_{t, x}(\lambda), g(\lambda)\right\rangle
$$

a.e. on $\Pi_{T}$. This implies that for a.e. $(t, x) \in \Pi_{T}$

$$
\int(\lambda-u)(g(\lambda)-g(u)) d \nu_{t, x}(\lambda)=\left\langle\nu_{t, x}(\lambda),(\lambda-u)(g(\lambda)-g(u))\right\rangle=0
$$

where $u=u(t, x)=\left\langle\nu_{t, x}(\lambda), \lambda\right\rangle$. Since $g(\lambda)$ increases the obtained relations can hold only if $g(\lambda) \equiv g(u)$ on cosupp $\nu_{t, x}$. Hence, for a.e. $(t, x) \in \Pi_{T} g(\lambda)$ is constant on $\operatorname{cosupp} \nu_{t, x}$. The proof is complete. 
Corollary 3.2. Assume that the sequence $u_{n}$ of weak solutions to approximate equations (1.6) weakly-* converges as $n \rightarrow \infty$ to a function $u=u(t, x) \in L^{\infty}\left(\Pi_{T}\right)$. Then $g\left(u_{n}\right) \underset{n \rightarrow \infty}{\rightarrow} g(u)$ in $L_{l o c}^{1}\left(\Pi_{T}\right)$ (strongly). Moreover, in the case when $g(u)$ is not constant on non-degenerate intervals, $u_{n} \underset{n \rightarrow \infty}{\rightarrow} u$ in $L_{l o c}^{1}\left(\Pi_{T}\right)$, and $u(t, x)$ is a weak solution of equation (1.1).

Proof. Extracting a subsequence, we can assume that the sequence $u_{n}$ converges to a measure-valued function $\nu_{t, x} \in \mathrm{MV}\left(\Pi_{T}\right)$. By Theorem 3.1 for a.e. $(t, x) \in \Pi_{T}$ $g(\lambda) \equiv g(u)$ on $\operatorname{cosupp} \nu_{t, x}$. Therefore, the image $g^{*} \nu_{t, x}$ coincides with the regular measure-valued function $\delta(\lambda-g(u(t, x)))$. As is easy to verify, $g^{*} \nu_{t, x}$ is a limit measurevalued function for the sequence $g\left(u_{n}\right)$. Since this measure-valued function is regular then by Theorem 2.3 we claim that $g\left(u_{n}\right) \rightarrow g(u)$ in $L_{l o c}^{1}\left(\Pi_{T}\right)$ as $n \rightarrow \infty$. Since the limit function $g(u)$ does not depend on the indicated above choice of a subsequence, we conclude that the original sequence $g\left(u_{n}\right)$ also strongly converges to $g(u)$.

In the case when the function $g(\lambda)$ is not constant on non-degenerate intervals the statement of Theorem 3.1 implies that the measure-valued function $\nu_{t, x}$ is regular itself: $\nu_{t, x}(\lambda)=\delta(\lambda-u(t, x))$. By Theorem 2.3, $u_{n} \rightarrow u$ strongly. Using again the fact that the limit function does not depend on the appropriate choice of a subsequence, we obtain that the original sequence $u_{n} \underset{n \rightarrow \infty}{\rightarrow} u$ in $L_{l o c}^{1}\left(\Pi_{T}\right)$. Passing to the limit as $n \rightarrow \infty$ in the distributional relations

$$
\left(u_{n}\right)_{t}+\left(\varphi_{n}\left(u_{n}\right)\right)_{x}-\left(g_{n}\left(u_{n}\right)\right)_{x x}=0,
$$

we readily derive that

$$
u_{t}+\varphi(u)_{x}-g(u)_{x x}=0 \text { in } \mathcal{D}^{\prime}\left(\Pi_{T}\right) .
$$

Further, by Lemma 2.2 with $a=-M, b=M$ for each $f=f(t, x) \in C_{0}^{\infty}\left(\Pi_{T}\right), f \geq 0$,

$$
\int_{\Pi_{T}}\left(\left(g_{n}\left(u_{n}\right)\right)_{x}\right)^{2} f d t d x \leq C_{f}
$$

where $C_{f}$ are constants independent of $n$. Thus, the sequence $\left(g_{n}\left(u_{n}\right)\right)_{x}$ is bounded in $L_{l o c}^{2}\left(\Pi_{T}\right)$ and after passage to a subsequence, if necessary, we can suppose that $\left(g_{n}\left(u_{n}\right)\right)_{x} \rightarrow q=q(t, x)$ as $n \rightarrow \infty$ weakly in $L_{l o c}^{2}\left(\Pi_{T}\right)$. As was already established, $g_{n}\left(u_{n}\right) \rightarrow g(u)$ in $L_{l o c}^{1}\left(\Pi_{T}\right)$ as $n \rightarrow \infty$. Passing to the limit as $n \rightarrow \infty$ in the relation

$$
\int_{\Pi_{T}} g_{n}\left(u_{n}\right) f_{x} d t d x=-\int_{\Pi_{T}}\left(g_{n}\left(u_{n}\right)\right)_{x} f d t d x, \quad f=f(t, x) \in C_{0}^{\infty}\left(\Pi_{T}\right),
$$

we arrive at the identity

$$
\int_{\Pi_{T}} g(u) f_{x} d t d x=-\int_{\Pi_{T}} q f d t d x, \quad f=f(t, x) \in C_{0}^{\infty}\left(\Pi_{T}\right),
$$

showing that $g(u)_{x}=q$. Hence $g(u)_{x} \in L_{l o c}^{2}\left(\Pi_{T}\right)$, as required. We conclude that $u=u(t, x)$ is a weak solution of (1.1). The proof is complete.

Now, we consider a bounded sequence $u_{n}=u_{n}(t, x)$ of entropy solutions to approximate equations (1.6). As above, we assume that $u_{n}$ converges as $n \rightarrow \infty$ to a bounded measure-valued function $\nu_{t, x}$ in the sense of relation (2.7). Let $M=$ $\sup \left\|u_{n}\right\|_{\infty}$. We are going to show that the flux function $\varphi(u)$ is affine on $\operatorname{cosupp} \nu_{t, x}$ for a.e. $(t, x) \in \Pi_{T}$. For this we need the following technical result. 
LEMma 3.3. Assume that $\nu$ is a finite nonnegative Borel measure on $\mathbb{R},[a, b]=$ $\operatorname{cosupp} \nu ; H(u) \in C(\mathbb{R})$, and for each $k \in(a, b)$

$$
\int(H(\lambda)-H(k)) \operatorname{sign}^{+}(\lambda-k) d \nu(\lambda)=0
$$

where $\operatorname{sign}^{+}(\lambda)=(1+\operatorname{sign} \lambda) / 2$ is the Heaviside function. Then $H(u) \equiv$ const on $[a, b]$.

The proof of this lemma given in [15, Lemma 2.3] can be simplified, and we put the revised proof below.

Proof. First, observe that by continuity of $H(\lambda)$ equality (3.3) holds for each $k \in[a, b]$. Since $H(u)$ is continuous there exist such $k_{1}, k_{2} \in[a, b]$ that

$$
H\left(k_{1}\right)=H_{-} \doteq \min _{u \in[a, b]} H(u), \quad H\left(k_{2}\right)=H_{+} \doteq \max _{u \in[a, b]} H(u) .
$$

Then it follows from (3.3) with $k=k_{1}, k_{2}$ that $H(b)=H_{-}=H_{+}$. Indeed, assuming that $H(b) \neq H_{-}$, we claim that $k_{1}<b$ and the nonnegative function $(H(\lambda)-$ $\left.H\left(k_{1}\right)\right) \operatorname{sign}^{+}\left(\lambda-k_{1}\right)$ is strictly positive in some neighborhood $(b-\delta, b]$ of the point $b$. Since $\nu((b-\delta, b])>0$ we conclude that the integral in equality (3.3) with $k=k_{1}$ is positive, which contradicts to this equality. Hence, $H(b)=H_{-}$. By the similar reasons, using (3.3) with $k=k_{2}$, we claim that $H(b)=H_{+}$. Evidently, the equality $H_{-}=H_{+}$can hold only if $H(u) \equiv$ const $=H(b)$ on the segment $[a, b]$. The proof is complete.

Denote by $\mathfrak{I}$ the set of segments $[a, b] \subset[-M, M]$ such that $a \leq b$ and $g(u)$ is constant on $[a, b]$ (i.e., $g(b)=g(a)$ ). Observe that by Theorem 3.1 for a.e. $(t, x) \in \Pi_{T}$ $\operatorname{cosupp} \nu_{t, x} \in \mathfrak{I}$. Now, we fix $[a, b] \in \mathfrak{I}$ such that $a<b$. Since the function $g(u)$ is defined up to an additive constant we may assume, without loss of generality, that $g(u) \equiv 0$ on $[a, b]$. Let, as above, $s_{a, b}(u)=\max (a, \min (b, u))$ be the cut-off function. We consider the sequence $v_{n}=s_{a, b}\left(u_{n}\right), n \in \mathbb{N}$. This sequence converges as $n \rightarrow \infty$ to the measure-valued function $\tilde{\nu}_{t, x}=s_{a, b}^{*} \nu_{t, x}$. Evidently, $\operatorname{cosupp} \tilde{\nu}_{t, x}=$ $s_{a, b}\left(\operatorname{cosupp} \nu_{t, x}\right)$

Proposition 3.4. For a.e. $(t, x) \in \Pi_{T}$ the function $\varphi(u)$ is affine on cosupp $\tilde{\nu}_{t, x}$.

Proof. We denote $u^{+}=\max (u, 0), u^{-}=\max (-u, 0)$. Let $\operatorname{sign}^{+}(u)=(\operatorname{sign} u)^{+}$ be the Heaviside function and $\operatorname{sign}^{-}(u)=-\operatorname{sign}^{+}(-u)$. We observe that for $k \in[a, b]$

$$
\begin{array}{r}
\left(v_{n}-k\right)^{+}=s_{k, b}\left(u_{n}\right)-k= \\
\left(\left|u_{n}-k\right|-\left|u_{n}-b\right|+b-k\right) / 2, \quad \operatorname{sign}^{+}\left(v_{n}-k\right)\left(\varphi_{n}\left(v_{n}\right)-\varphi_{n}(k)\right)= \\
\varphi_{n}\left(s_{k, b}\left(u_{n}\right)\right)-\varphi_{n}(k)=\left(\operatorname{sign}\left(u_{n}-k\right)\left(\varphi_{n}\left(u_{n}\right)-\varphi_{n}(k)\right)-\right. \\
\left.\operatorname{sign}\left(u_{n}-b\right)\left(\varphi_{n}\left(u_{n}\right)-\varphi_{n}(b)\right)+\varphi_{n}(b)-\varphi_{n}(k)\right) / 2 \\
\left(v_{n}-k\right)^{-}=k-s_{a, k}\left(u_{n}\right)= \\
\left(\left|u_{n}-k\right|-\left|u_{n}-a\right|+k-a\right) / 2, \quad \operatorname{sign}^{-}\left(v_{n}-k\right)\left(\varphi_{n}\left(v_{n}\right)-\varphi_{n}(k)\right)= \\
\varphi_{n}(k)-\varphi_{n}\left(s_{a, k}\left(u_{n}\right)\right)=\left(\operatorname{sign}\left(u_{n}-k\right)\left(\varphi_{n}\left(u_{n}\right)-\varphi_{n}(k)\right)-\right. \\
\left.\operatorname{sign}\left(u_{n}-a\right)\left(\varphi_{n}\left(u_{n}\right)-\varphi_{n}(a)\right)+\varphi_{n}(k)-\varphi_{n}(a)\right) / 2
\end{array}
$$

Since $u_{n}$ is an entropy solution of (1.6) then by Lemma 2.1 for each $k \in[a, b]$

$\left|u_{n}-k\right|_{t}+\left[\operatorname{sign}\left(u_{n}-k\right)\left(\varphi_{n}\left(u_{n}\right)-\varphi_{n}(k)\right)\right]_{x}-\left|g_{n}\left(u_{n}\right)-g_{n}(k)\right|_{x x}=-\mu_{k}^{n}$ in $\mathcal{D}^{\prime}\left(\Pi_{T}\right)$, 
where, in view of $(2.2), \mu_{k}^{n}$ are bounded sequences in the space of $\mathrm{M}_{l o c}\left(\Pi_{T}\right)$ of locally finite Borel measures in $\Pi_{T}$. We denote

$\eta_{k}^{ \pm}(u)=(u-k)^{ \pm}, \quad \psi_{k}^{ \pm}=\operatorname{sign}^{ \pm}(u-k)(\varphi(u)-\varphi(k)), \quad \psi_{k n}^{ \pm}=\operatorname{sign}^{ \pm}(u-k)\left(\varphi_{n}(u)-\varphi_{n}(k)\right)$.

Then, by identities (3.4), (3.5) and relation (3.6)

$$
\begin{array}{r}
\left(\eta_{k}^{+}\left(v_{n}\right)\right)_{t}+\left(\psi_{k}^{+}\left(v_{n}\right)\right)_{x}= \\
\left(\mu_{b}^{n}-\mu_{k}^{n}\right) / 2+\left(g_{n}\left(s_{k, b}\left(u_{n}\right)\right)\right)_{x x}+\left(\psi_{k}^{+}\left(v_{n}\right)-\psi_{k n}^{+}\left(v_{n}\right)\right)_{x}, \\
\left(\eta_{k}^{-}\left(v_{n}\right)\right)_{t}+\left(\psi_{k}^{-}\left(v_{n}\right)\right)_{x}= \\
\left(\mu_{a}^{n}-\mu_{k}^{n}\right) / 2-\left(g_{n}\left(s_{a, k}\left(u_{n}\right)\right)\right)_{x x}+\left(\psi_{k}^{-}\left(v_{n}\right)-\psi_{k n}^{-}\left(v_{n}\right)\right)_{x}
\end{array}
$$

in $\mathcal{D}^{\prime}\left(\Pi_{T}\right)$. Here we also take into account that

$$
\begin{array}{r}
\left(\left|g_{n}\left(u_{n}\right)-g(k)\right|-\left|g_{n}\left(u_{n}\right)-g_{n}(b)\right|\right) / 2=g_{n}\left(s_{k, b}\left(u_{n}\right)\right)+\text { const } \\
\left(\left|g_{n}\left(u_{n}\right)-g(k)\right|-\left|g_{n}\left(u_{n}\right)-g_{n}(a)\right|\right) / 2=-g_{n}\left(s_{a, k}\left(u_{n}\right)\right)+\text { const } .
\end{array}
$$

Denoting $\gamma_{k n}^{+}=\left(\mu_{b}^{n}-\mu_{k}^{n}\right) / 2, \gamma_{k n}^{-}=\left(\mu_{a}^{n}-\mu_{k}^{n}\right) / 2, L_{k n}^{+}=\psi_{k}^{+}\left(v_{n}\right)-\psi_{k n}^{+}\left(v_{n}\right)+$ $\left(g_{n}\left(s_{k, b}\left(u_{n}\right)\right)\right)_{x}, L_{k n}^{-}=\psi_{k}^{-}\left(v_{n}\right)-\psi_{k n}^{-}\left(v_{n}\right)-\left(g_{n}\left(s_{a, k}\left(u_{n}\right)\right)\right)_{x}$, we can rewrite $(3.7)$, (3.8) in the form

$$
\left(\eta_{k}^{ \pm}\left(v_{n}\right)\right)_{t}+\left(\psi_{k}^{ \pm}\left(v_{n}\right)\right)_{x}=\gamma_{k n}^{ \pm}+\left(L_{k n}^{ \pm}\right)_{x}
$$

By the condition of uniform on $[a, b]$ convergence $\varphi_{n}(u) \rightarrow \varphi(u)$ we have $\psi_{k}^{ \pm}\left(v_{n}\right)-$ $\psi_{k n}^{ \pm}\left(v_{n}\right) \rightarrow 0$ as $n \rightarrow \infty$ in $L_{l o c}^{1}\left(\Pi_{T}\right)$ (and even in $L^{\infty}\left(\Pi_{T}\right)$ ). Further, by Lemma 2.2 for each $f=f(t, x) \in C_{0}^{\infty}\left(\Pi_{T}\right), f \geq 0$

$$
\begin{array}{r}
\int_{\Pi_{T}}\left[\left(\left(g_{n}\left(s_{k, b}\left(u_{n}\right)\right)\right)_{x}\right)^{2}+\left(\left(g_{n}\left(s_{a, k}\left(u_{n}\right)\right)\right)_{x}\right)^{2}\right] f d t d x= \\
\int_{a \leq u_{n}(t, x) \leq b}\left(g_{n}\left(u_{n}\right)_{x}\right)^{2} f d t d x \leq C_{f} \max _{a \leq u \leq b}\left|g_{n}(u)\right|,
\end{array}
$$

where $C_{f}$ is some constant depending only on $f$. By our assumptions, $g_{n}(u) \rightarrow$ $g(u) \equiv 0$ as $n \rightarrow \infty$ uniformly on $[a, b]$ and in view of (3.10) we see that the sequences $\left(g_{n}\left(s_{k, b}\left(u_{n}\right)\right)\right)_{x},\left(g_{n}\left(s_{a, k}\left(u_{n}\right)\right)\right)_{x} \rightarrow 0$ as $n \rightarrow \infty$ in $L_{l o c}^{2}\left(\Pi_{T}\right)$. Hence, $L_{k n}^{ \pm} \underset{n \rightarrow \infty}{\rightarrow} 0$ in $L_{l o c}^{2}\left(\Pi_{T}\right)$. This implies that the sequences of distributions $\left(L_{k n}^{ \pm}\right)_{x} \underset{n \rightarrow \infty}{\rightarrow} 0$ in the Sobolev space $W_{2, l o c}^{-1}\left(\Pi_{T}\right)$, which is a locally convex space of distributions $l=l(t, x)$ such that $l h$ belongs to the Sobolev space $W_{2}^{-1}$ (recall that $W_{2}^{-1}$ is the dual space to the Sobolev space $\left.W_{2}^{1}=W_{2}^{1}\left(\mathbb{R}^{2}\right)\right)$ for all $h=h(t, x) \in C_{0}^{\infty}\left(\Pi_{T}\right)$. The topology in $W_{2, l o c}^{-1}\left(\Pi_{T}\right)$ is generated by the family of seminorms $l \rightarrow\|l h\|_{W_{2}^{-1}}, h \in C_{0}^{\infty}\left(\Pi_{T}\right)$.

Since the sequences $\gamma_{k n}^{ \pm}$are bounded in $\mathrm{M}_{l o c}\left(\Pi_{T}\right)$ then, by Murat interpolation lemma [12] (also see [19, Lemma 28]), the sequences (3.9) are pre-compact in $W_{2, l o c}^{-1}\left(\Pi_{T}\right)$ for each $k \in[a, b]$. By Tartar-Murat compensated compactness $[11,19]$ (see also [17, Corollary 3.2]) the quadratic functional $q(\bar{\lambda})=\left(\lambda_{1} \lambda_{4}-\lambda_{2} \lambda_{3}\right), \bar{\lambda}=$ $\left(\lambda_{1}, \lambda_{2}, \lambda_{3}, \lambda_{4}\right)$, is weakly continuous on the sequences $\left(\eta_{k}^{+}\left(v_{n}\right), \psi_{k}^{+}\left(v_{n}\right), \eta_{k}^{-}\left(v_{n}\right), \psi_{l}^{-}\left(v_{n}\right)\right)$ for all $k, l \in[a, b]$. By (2.7) this can be written as the following commutation relation: for a.e. $(t, x) \in \Pi_{T}$

$$
\begin{array}{r}
\left\langle\tilde{\nu}_{t, x}(\lambda), \eta_{k}^{+}(\lambda) \psi_{l}^{-}(\lambda)-\eta_{l}^{-}(\lambda) \psi_{k}^{+}(\lambda)\right\rangle= \\
\left\langle\tilde{\nu}_{t, x}(\lambda), \eta_{k}^{+}(\lambda)\right\rangle\left\langle\tilde{\nu}_{t, x}(\lambda), \psi_{l}^{-}(\lambda)\right\rangle-\left\langle\tilde{\nu}_{t, x}(\lambda), \eta_{l}^{-}(\lambda)\right\rangle\left\langle\tilde{\nu}_{t, x}(\lambda), \psi_{k}^{+}(\lambda)\right\rangle .
\end{array}
$$


It is clear that (3.11) holds for $(t, x) \in P$, where $P$ is a set of common Lebesgue point of the functions $(t, x) \rightarrow\left\langle\nu_{t, x}(\lambda), p(\lambda)\right\rangle, p(\lambda) \in C(\mathbb{R})$. Since the space $C(\mathbb{R})$ is separable, we see that $P \subset \Pi_{T}$ is a set of full measure. Noticing that

$$
\left\langle\tilde{\nu}_{t, x}(\lambda), p(\lambda)\right\rangle=\left\langle\nu_{t, x}(\lambda), p\left(s_{a, b}(\lambda)\right)\right\rangle,
$$

we see that points of $P$ are Lebesgue points of all functions $\left\langle\tilde{\nu}_{t, x}(\lambda), p(\lambda)\right\rangle, p(\lambda) \in$ $C(\mathbb{R})$. We fix $(t, x) \in P, \tilde{\nu}=\tilde{\nu}_{t, x}$, and assume that the segment $\left[a_{1}, b_{1}\right]=\operatorname{cosupp} \tilde{\nu}$ is not trivial, i.e., $a_{1}<b_{1}$. Then it follows from (3.11) that for each $k, l \in\left(a_{1}, b_{1}\right)$ such that $l<k$

$$
\left\langle\tilde{\nu}(\lambda), \eta_{k}^{+}(\lambda)\right\rangle\left\langle\tilde{\nu}(\lambda), \psi_{l}^{-}(\lambda)\right\rangle-\left\langle\tilde{\nu}(\lambda), \eta_{l}^{-}(\lambda)\right\rangle\left\langle\tilde{\nu}(\lambda), \psi_{k}^{+}(\lambda)\right\rangle=0
$$

because, evidently, $\eta_{k}^{+}(\lambda) \psi_{l}^{-}(\lambda)=\eta_{l}^{-}(\lambda) \psi_{k}^{+}(\lambda) \equiv 0$. Since $\left[a_{1}, b_{1}\right]$ is the minimal segment containing $\operatorname{supp} \tilde{\nu}$ then

$$
\begin{gathered}
\left\langle\tilde{\nu}(\lambda), \eta_{k}^{+}(\lambda)\right\rangle=\int(\lambda-k)^{+} d \tilde{\nu}(\lambda)>0 \\
\left\langle\tilde{\nu}(\lambda), \eta_{l}^{-}(\lambda)\right\rangle=\int(\lambda-l)^{-} d \tilde{\nu}(\lambda)>0
\end{gathered}
$$

and (3.12) implies that for each $l, k \in\left(a_{1}, b_{1}\right), l<k$

$$
I_{-}(l) \doteq \frac{\left\langle\tilde{\nu}(\lambda), \psi_{l}^{-}(\lambda)\right\rangle}{\left\langle\tilde{\nu}(\lambda), \eta_{l}^{-}(\lambda)\right\rangle}=I_{+}(k)=\frac{\left\langle\tilde{\nu}(\lambda), \psi_{k}^{+}(\lambda)\right\rangle}{\left\langle\tilde{\nu}(\lambda), \eta_{k}^{+}(\lambda)\right\rangle} .
$$

Clearly, this can hold only if $I_{-}(l)=I_{+}(k)=C$, where $C=$ const. In particular, $I_{+}(k)=C$, which implies that

$\int \operatorname{sign}^{+}(\lambda-k)(\varphi(\lambda)-C \lambda-(\varphi(k)-C k)) d \tilde{\nu}(\lambda)=\left\langle\tilde{\nu}(\lambda), \psi_{k}^{+}(\lambda)\right\rangle-C\left\langle\tilde{\nu}(\lambda), \eta_{k}^{+}(\lambda)\right\rangle=0$

for all $k \in\left(a_{1}, b_{1}\right)$. By Lemma 3.3, applied to the function $H(\lambda)=\varphi(\lambda)-C \lambda$, we conclude that $\varphi(\lambda)-C \lambda=$ const on $\left[a_{1}, b_{1}\right]$, that is, $\varphi(\lambda)$ is affine on $\left[a_{1}, b_{1}\right]=$ $\operatorname{cosupp} \tilde{\nu}_{t, x}$. In the case $a_{1}=b_{1}$ this statement is trivially fulfilled. To conclude the proof, it only remains to see that $(t, x) \in P$ is arbitrary.

Now we can prove the following important result.

THEOREM 3.5. For a.e. $(t, x) \in \Pi_{T} \quad g(\lambda)$ is constant and $\varphi(\lambda)$ is affine on $\operatorname{cosupp} \nu_{t, x}$. In particular, the limit function $u(t, x)$ is a weak solution of (1.1).

Proof. Let $P \subset \Pi_{T}$ be defined as in the proof of Proposition 3.4. We fix $(t, x) \in$ $P$ and show that $g(\lambda)$ is constant and $\varphi(\lambda)$ is affine on $[a, b]=\operatorname{cosupp} \nu_{t, x}$. This is evident if $a=b$. Hence assume that $a<b$ and notice that, as follows from Theorem 3.1, $g(\lambda)=$ const on $[a, b]$. Remark that $\operatorname{supp} \nu_{t, x} \subset[a, b]$. Therefore, $s_{a, b}^{*} \nu_{t, x}=\nu_{t, x}$. Hence, by Proposition $3.4, \varphi(\lambda)$ is affine on $[a, b]$. It remains only to prove that $u(t, x)$ is a weak solution of (1.1). By limit relation (2.7)

$$
\varphi\left(u_{n}\right) \underset{n \rightarrow \infty}{\rightarrow}\left\langle\nu_{t, x}(\lambda), \varphi(\lambda)\right\rangle=\varphi\left(\left\langle\nu_{t, x}(\lambda), \lambda\right\rangle\right)=\varphi(u(t, x)),
$$

where we take into account that $\varphi(\lambda)$ is affine on $\operatorname{cosupp} \nu_{t, x}$. By Corollary 3.2 $g\left(u_{n}\right) \underset{n \rightarrow \infty}{\rightarrow} g(u)$ in $L_{l o c}^{1}\left(\Pi_{T}\right)$. It directly follows from the above limit relations that 
$\varphi_{n}\left(u_{n}\right) \underset{n \rightarrow \infty}{\rightarrow} \varphi(u)$ weakly-* in $L^{\infty}\left(\Pi_{T}\right), g_{n}\left(u_{n}\right) \underset{n \rightarrow \infty}{\rightarrow} g(u)$ in $L_{l o c}^{1}\left(\Pi_{T}\right)$. Therefore, we can pass to the limit as $n \rightarrow \infty$ in the identity

$$
\int_{\Pi_{T}}\left\{u_{n} f_{t}+\varphi_{n}\left(u_{n}\right) f_{x}-g_{n}\left(u_{n}\right) f_{x x}\right\} d t d x, \quad f=f(t, x) \in C_{0}^{\infty}\left(\Pi_{T}\right)
$$

and arrive at the relation

$$
\int_{\Pi_{T}}\left\{u f_{t}+\varphi(u) f_{x}-g(u) f_{x x}\right\} d t d x, \quad \forall f=f(t, x) \in C_{0}^{\infty}\left(\Pi_{T}\right),
$$

showing that $u=u(t, x)$ satisfies $(1.1)$ in $\mathcal{D}^{\prime}(\Pi)$. By Lemma 2.2 the sequence $\left(g_{n}\left(u_{n}\right)\right)_{x}$ is bounded in $L_{l o c}^{2}\left(\Pi_{T}\right)$. As shown in the proof of Corollary 3.2, this implies that $g(u)_{x} \in L_{l o c}^{2}\left(\Pi_{T}\right)$. Hence, $u(t, x)$ is a weak solution of (1.1).

COROLlary 3.6. Assume that $g(u)$ is not constant and $\varphi(u)$ is not affine simultaneously on nondegenerate intervals. Then the sequence $u_{n} \rightarrow u$ as $n \rightarrow \infty$ in $L_{l o c}^{1}\left(\Pi_{T}\right)$, and the limit function is an entropy solution of (1.1).

Proof. Passing to a subsequence, as usual, we may suppose that $u_{n}$ converges to a bounded measure-valued function $\nu_{t, x}$ in the sense of (2.7). By Theorem 3.5 and our assumption we see that $\operatorname{cosupp} \nu_{t, x}$ is a point $\{u(t, x)\}$ for a.e. $(t, x) \in \Pi_{T}$. This means that $\nu_{t, x}(\lambda)=\delta(\lambda-u(t, x))$ a.e. on $\Pi_{T}$. By Theorem 2.3 we claim that $u_{n} \rightarrow u$ as $n \rightarrow \infty$ in $L_{l o c}^{1}\left(\Pi_{T}\right)$. Since the limit function does not depend on the choice of a subsequence, we see that the above limit relation remains valid for the original sequence. Observe that by Theorem $3.5 u(t, x)$ is a weak solution of (1.1). To prove that $u(t, x)$ is an entropy solution, we only need to pass to the limit as $n \rightarrow \infty$ in the entropy conditions

$$
\int_{\Pi_{T}}\left\{\left|u_{n}-k\right| f_{t}+\left[\operatorname{sign}\left(u_{n}-k\right)\left(\varphi_{n}\left(u_{n}\right)-\varphi_{n}(k)\right)\right] f_{x}+\left|g_{n}\left(u_{n}\right)-g_{n}(k)\right| f_{x x}\right\} d t d x \geq 0
$$

with $f=f(t, x) \in C_{0}^{\infty}\left(\Pi_{T}\right), f \geq 0$, and derive (1.5).

\section{口}

Remark 1. The statement of Corollary 3.6 (the strong precompactness property) also follows from localization principles for $H$-measures corresponding to approximate sequences of entropy solution, see [16, 8] for the case of general multidimensional parabolic and elliptic equations.

4. Main result. Theorem 3.5 shows that a weak limit of entropy solutions to equation (1.1) is a weak solution of this equation. But we are going to establish a stronger result asserting that this weak limit is actually an entropy solution. For this we have to analyze the properties of the limit measure-valued function $\nu_{t, x}$ more precisely. Namely, we now utilize the entropy relations (1.5): for each $k \in \mathbb{R}$ and any non-negative test function $f=f(t, x) \in C_{0}^{\infty}\left(\Pi_{T}\right)$

$$
\int_{\Pi_{T}}\left\{\left|u_{n}-k\right| f_{t}+\left[\operatorname{sign}\left(u_{n}-k\right)\left(\varphi_{n}\left(u_{n}\right)-\varphi_{n}(k)\right)\right] f_{x}+\left|g_{n}\left(u_{n}\right)-g_{n}(k)\right| f_{x x}\right\} d t d x \geq 0 .
$$

Passing in this relation to the limit as $n \rightarrow \infty$ and using (2.7), we obtain that

$\int_{\Pi_{T}}\left\{\left\langle\nu_{t, x}(\lambda),|\lambda-k|\right\rangle f_{t}+\left\langle\nu_{t, x}(\lambda), \operatorname{sign}(\lambda-k)(\varphi(\lambda)-\varphi(k))\right\rangle f_{x}+|g(u)-g(k)| f_{x x}\right\} d t d x \geq 0$. 
Here we also use the fact that $g(\lambda) \equiv g(u)$ on $\operatorname{supp} \nu_{t, x}$. Recall that $u=u(t, x)=$ $\left\langle\nu_{t, x}(\lambda), \lambda\right\rangle$. Relation (4.1) means that for each $k \in \mathbb{R}$

$$
\left(\left\langle\nu_{t, x}(\lambda),|\lambda-k|\right\rangle\right)_{t}+\left(\left\langle\nu_{t, x}(\lambda), \operatorname{sign}(\lambda-k)(\varphi(\lambda)-\varphi(k))\right\rangle\right)_{x}-(|g(u)-g(k)|)_{x x} \leq 0
$$

in $\mathcal{D}^{\prime}\left(\Pi_{T}\right)$.

Following [6], we call such $\nu_{t, x}$ a measure-valued entropy solution of (1.1).

By Theorem $3.5 u=u(t, x)$ is a weak solution of (1.1). Therefore, $u_{t}+(\varphi(u)-$ $\left.g(u)_{x}\right)_{x}=0$ and the vector field $\left(u, \varphi(u)-g(u)_{x}\right) \in \mathbb{R}^{2}$ is divergence-free. By the known results on the existence of weak normal traces (see, for instance, [3]) there exists a weak trace $u_{0}(x) \in L^{\infty}(\mathbb{R})$ of $u(t, x)$, that is, ess $\lim _{t \rightarrow 0+} u(t, \cdot)=u_{0}$ in the weak-* topology of $L^{\infty}(\mathbb{R})$. It is clear that $\left\|u_{0}\right\|_{\infty} \leq M$. As was shown in [10], there exists a unique entropy solution $u=v(t, x)$ to the Cauchy problem for equation (1.1) with initial data $u_{0}(x)$. Recall that the initial condition is understood in the sense of strong trace relation

$$
\underset{t \rightarrow 0+}{\operatorname{ess}} \lim _{t \rightarrow} v(t, \cdot)=u_{0} \text { in } L_{l o c}^{1}(\mathbb{R}) .
$$

Notice that by the comparison principle (see [10]) $\|v\|_{\infty}=\left\|u_{0}\right\|_{\infty} \leq M$. We shall prove that $u=v$ a.e. on $\Pi_{T}$. For that we firstly adapt the Carrillo variant of the doubling variable method (cf. [2]) to obtain the measure-valued analog of the so-called Kato inequality.

Let $s_{\varepsilon}(u)=s_{-1,1}(u / \varepsilon), \varepsilon>0$, be a regularization of $\operatorname{sign} u$, and let $E$ be a set of discontinuity points of the increasing function $g_{0}^{-1}(v)$.

Lemma 4.1. Let $u=u(t, x)$ be a weak solution of equation (1.1). Suppose that $g(v) \notin E$ and $\xi=\xi(t, x) \in C_{0}^{\infty}\left(\Pi_{T}\right), \xi \geq 0$. Then

$$
\begin{array}{r}
\int_{\Pi_{T}} \operatorname{sign}(u-v)\left\{(u-v) \xi_{t}+\left(\varphi(u)-\varphi(v)-g(u)_{x}\right) \xi_{x}\right\} d t d x= \\
\lim _{\varepsilon \rightarrow 0} \int_{\Pi_{T}}\left(g(u)_{x}\right)^{2} s_{\varepsilon}^{\prime}(g(u)-g(v)) \xi d t d x .
\end{array}
$$

Proof. Let $H_{\varepsilon}(u)=s_{0,1}(u / \varepsilon)$. Then, by [2, Lemma 5]

$$
\begin{array}{r}
\int_{\Pi_{T}} \operatorname{sign}^{+}(u-v)\left\{(u-v) \xi_{t}+\left(\varphi(u)-\varphi(v)-g(u)_{x}\right) \xi_{x}\right\} d t d x= \\
\lim _{\varepsilon \rightarrow 0} \int_{\Pi_{T}}\left(g(u)_{x}\right)^{2} H_{\varepsilon}^{\prime}(g(u)-g(v)) \xi d t d x \\
\int_{\Pi_{T}} \operatorname{sign}^{-}(u-v)\left\{(u-v) \xi_{t}+\left(\varphi(u)-\varphi(v)-g(u)_{x}\right) \xi_{x}\right\} d t d x= \\
\lim _{\varepsilon \rightarrow 0} \int_{\Pi_{T}}\left(g(u)_{x}\right)^{2} H_{\varepsilon}^{\prime}(g(v)-g(u)) \xi d t d x .
\end{array}
$$

Putting these two relation together, we arrive at (4.3).

Proposition 4.2. The following relation (the Kato inequality) holds in $\mathcal{D}^{\prime}\left(\Pi_{T}\right)$

$$
\begin{array}{r}
\frac{\partial}{\partial t}\left\langle\nu_{t, x}(\lambda),|\lambda-v(t, x)|\right\rangle+\frac{\partial}{\partial x}\left\langle\nu_{t, x}(\lambda), \operatorname{sign}(\lambda-v(t, x))(\varphi(\lambda)-\varphi(v(t, x)))\right\rangle \\
-\frac{\partial^{2}}{\partial x^{2}}|u(t, x)-v(t, x)| \leq 0 .
\end{array}
$$


Proof. Let us consider two different pairs of variables $(t, x)$ and $(s, y)$ in $\Pi_{T}$. We set $k=v(s, y)$ in (4.2). Applying this relation to a non-negative test function $\xi=\xi(t, x ; s, y) \in C_{0}^{\infty}\left(\Pi_{T} \times \Pi_{T}\right)$, we obtain that for every $(s, y) \in \Pi_{T}$

$$
\begin{array}{r}
\int_{\Pi_{T}}\left\{\left\langle\nu_{t, x}(\lambda),|\lambda-v|\right\rangle \xi_{t}+\right. \\
\left.\left\langle\nu_{t, x}(\lambda), \operatorname{sign}(\lambda-v)(\varphi(\lambda)-\varphi(v))\right\rangle \xi_{x}-(|g(u)-g(v)|)_{x} \xi_{x}\right\} d t d x \geq 0,
\end{array}
$$

where $u=u(t, x), v=v(s, y)$. As in the proof of Lemma 2.2, let $E$ be a set of discontinuity points of the increasing function $g_{0}^{-1}(v)$. We introduce the sets

$$
Q_{1}=\left\{(t, x) \mid u(t, x) \in g^{-1}(E)\right\}, Q_{2}=\left\{(s, y) \mid v(s, y) \in g^{-1}(E)\right\}
$$

Since $E$ is at most countable then $g(u)_{x}=0$ a.e. on $Q_{1}$ and $g(v)_{y}=0$ a.e. on $Q_{2}$. Also notice that by the chain rule

$$
(|g(u)-g(v)|)_{x}=\operatorname{sign}(u-v) g(u)_{x}, \quad(|g(u)-g(v)|)_{y}=-\operatorname{sign}(u-v)(g(v))_{y} .
$$

If $(s, y) \notin Q_{2}$ then $g(v) \notin E$ and $\operatorname{sign}(\lambda-v)=$ const on the segment $[a, b]=\operatorname{cosupp} \nu_{t, x}$ for a.e. $(t, x) \in \Pi_{T}$. Indeed, by Theorem 3.1, for a.e. $(t, x) \in \Pi_{T}$ either $a=b$ or $[a, b] \in g^{-1}(E)$ (then $\left.v \notin[a, b]\right)$. Therefore, in the case $(s, y) \notin Q_{2}$ for a.e. $(t, x) \in \Pi_{T}$

$\left\langle\nu_{t, x}(\lambda),|\lambda-v|\right\rangle=|u-v|,\left\langle\nu_{t, x}(\lambda), \operatorname{sign}(\lambda-v)(\varphi(\lambda)-\varphi(v))\right\rangle=\operatorname{sign}(u-v)(\varphi(u)-\varphi(v))$.

Integrating (4.5) with respect to $(s, y)$ and taking into account (4.6), (4.7) and Lemma 4.1, we deduce that

$$
\begin{array}{r}
\int_{\Pi_{T} \times \Pi_{T}}\left\{\left\langle\nu_{t, x}(\lambda),|\lambda-v|\right\rangle \xi_{t}+\right. \\
\left.\left\langle\nu_{t, x}(\lambda), \operatorname{sign}(\lambda-v)(\varphi(\lambda)-\varphi(v))\right\rangle \xi_{x}-(|g(u)-g(v)|)_{x} \xi_{x}\right\} d t d x d s d y \geq \\
\int_{\Pi_{T} \times\left(\Pi_{T} \backslash Q_{2}\right)} \operatorname{sign}(u-v)\left\{(u-v) \xi_{t}+\left(\varphi(u)-\varphi(v)-g(u)_{x}\right) \xi_{x}\right\} d t d x d s d y= \\
\lim _{\varepsilon \rightarrow 0} \int_{\Pi_{T} \times\left(\Pi_{T} \backslash Q_{2}\right)}\left(g(u)_{x}\right)^{2} s_{\varepsilon}^{\prime}(g(u)-g(v)) \xi d t d x d s d y= \\
\lim _{\varepsilon \rightarrow 0} \int_{\left(\Pi_{T} \backslash Q_{1}\right) \times\left(\Pi_{T} \backslash Q_{2}\right)}\left(g(u)_{x}\right)^{2} s_{\varepsilon}^{\prime}(g(u)-g(v)) \xi d t d x d s d y
\end{array}
$$

Since $v=v(s, y)$ is a weak solution of (1.1) then for each $\lambda \in \mathbb{R}$

$$
\int_{\Pi_{T}}\left\{|v-\lambda| \xi_{s}+\operatorname{sign}(v-\lambda)(\varphi(v)-\varphi(\lambda)) \xi_{y}-(|g(v)-g(\lambda)|)_{y} \xi_{y}\right\} d s d y \geq 0
$$

Integrating this relation firstly with respect to the measure $\nu_{t, x}(\lambda)$ and after with 
respect to $(t, x)$, we find that

$$
\begin{array}{r}
\int_{\Pi_{T} \times \Pi_{T}}\left\{\left\langle\nu_{t, x}(\lambda),|\lambda-v|\right\rangle \xi_{s}+\right. \\
\left.\left\langle\nu_{t, x}(\lambda), \operatorname{sign}(\lambda-v)(\varphi(\lambda)-\varphi(v))\right\rangle \xi_{y}-(|g(u)-g(v)|)_{y} \xi_{y}\right\} d t d x d s d y \geq \\
\int_{\left(\Pi_{T} \backslash Q_{1}\right) \times \Pi_{T}} \operatorname{sign}(u-v)\left\{(u-v) \xi_{s}+\left(\varphi(u)-\varphi(v)+g(v)_{y}\right) \xi_{y}\right\} d t d x d s d y= \\
\lim _{\varepsilon \rightarrow 0} \int_{\left(\Pi_{T} \backslash Q_{1}\right) \times \Pi_{T}}\left(g(v)_{y}\right)^{2} s_{\varepsilon}^{\prime}(g(v)-g(u)) \xi d t d x d s d y= \\
\lim _{\varepsilon \rightarrow 0} \int_{\left(\Pi_{T} \backslash Q_{1}\right) \times\left(\Pi_{T} \backslash Q_{2}\right)}\left(g(v)_{y}\right)^{2} s_{\varepsilon}^{\prime}(g(u)-g(v)) \xi d t d x d s d y
\end{array}
$$

Here we take into account that in view of Theorem $3.1 \nu_{t, x}=\delta(\lambda-u(t, x))$ for a.e. $(t, x) \in \Pi_{T} \backslash Q_{1}$. We also use Lemma 4.1, the second equality in (4.6), and the fact that $s_{\varepsilon}^{\prime}$ is an even function.

Now, since $g(u)_{x}$ does not depend on $(s, y)$, we have

$$
\int_{\Pi_{T}} g(u)_{x}\left(s_{\varepsilon}(g(u)-g(v)) \xi\right)_{y} d s d y=0
$$

which implies the relation

$$
\int_{\Pi_{T}} s_{\varepsilon}(g(u)-g(v)) g(u)_{x} \xi_{y} d s d y=\int_{\Pi_{T}} g(u)_{x} g(v)_{y} s_{\varepsilon}^{\prime}(g(u)-g(v)) \xi d s d y .
$$

Passing in this relation to the limit as $\varepsilon \rightarrow 0$, we deduce that

$$
\int_{\Pi_{T}} \operatorname{sign}(g(u)-g(v)) g(u)_{x} \xi_{y} d s d y=\lim _{\varepsilon \rightarrow 0} \int_{\Pi_{T}} g(u)_{x} g(v)_{y} s_{\varepsilon}^{\prime}(g(u)-g(v)) \xi d s d y .
$$

Integrating this equality over $(t, x) \in \Pi_{T}$, we get

$$
\begin{array}{r}
\int_{\Pi_{T} \times \Pi_{T}} \operatorname{sign}(g(u)-g(v)) g(u)_{x} \xi_{y} d t d x d s d y= \\
\lim _{\varepsilon \rightarrow 0} \int_{\Pi_{T} \times \Pi_{T}} g(u)_{x} g(v)_{y} s_{\varepsilon}^{\prime}(g(u)-g(v)) \xi d t d x d s d y .
\end{array}
$$

Noticing that $\operatorname{sign}(g(u)-g(v))=\operatorname{sign}(u-v)$ if $(t, x) \notin Q_{1}$ while $g(u)_{x}=0$ for a.e. $(t, x) \in Q_{1}$ and that $g(v)_{y}=0$ for a.e. $(s, y) \in Q_{2}$, we can transform the above relation as follows

$$
\begin{array}{r}
\int_{\Pi_{T} \times \Pi_{T}} \operatorname{sign}(u-v) g(u)_{x} \xi_{y} d t d x d s d y= \\
\lim _{\varepsilon \rightarrow 0} \int_{\left(\Pi_{T} \backslash Q_{1}\right) \times\left(\Pi_{T} \backslash Q_{2}\right)} g(u)_{x} g(v)_{y} s_{\varepsilon}^{\prime}(g(u)-g(v)) \xi d t d x d s d y .
\end{array}
$$

Similarly, from the equality

$$
\int_{\Pi_{T}} g(v)_{y}\left(s_{\varepsilon}(g(u)-g(v)) \xi\right)_{x} d t d x=0
$$


we deduce the relation

$$
\begin{array}{r}
-\int_{\Pi_{T} \times \Pi_{T}} \operatorname{sign}(u-v) g(v)_{y} \xi_{x} d t d x d s d y= \\
\lim _{\varepsilon \rightarrow 0} \int_{\left(\Pi_{T} \backslash Q_{1}\right) \times\left(\Pi_{T} \backslash Q_{2}\right)} g(u)_{x} g(v)_{y} s_{\varepsilon}^{\prime}(g(u)-g(v)) \xi d t d x d s d y .
\end{array}
$$

By subtracting (4.10) and (4.11) from the sum of (4.8) and (4.9), we find that

$$
\begin{array}{r}
\int_{\Pi_{T} \times \Pi_{T}}\left\{\left\langle\nu_{t, x}(\lambda),|\lambda-v|\right\rangle\left(\xi_{t}+\xi_{s}\right)+\right. \\
\left\langle\nu_{t, x}(\lambda), \operatorname{sign}(\lambda-v)(\varphi(\lambda)-\varphi(v))\right\rangle\left(\xi_{x}+\xi_{y}\right)- \\
\left.\operatorname{sign}(u-v)\left(g(u)_{x}-g(v)_{y}\right)\left(\xi_{x}+\xi_{y}\right)\right\} d t d x d s d y \geq \\
\lim _{\varepsilon \rightarrow 0} \int_{\left(\Pi_{T} \backslash Q_{1}\right) \times\left(\Pi_{T} \backslash Q_{2}\right)}\left(g(u)_{x}-g(v)_{y}\right)^{2} s_{\varepsilon}^{\prime}(g(u)-g(v)) \xi d t d x d s d y \geq 0 .
\end{array}
$$

Notice that $\operatorname{sign}(u-v)\left(g(u)_{x}-g(v)_{y}\right)=(\partial / \partial x+\partial / \partial y)|g(u)-g(v)|$ and, integrating by parts, we obtain from (4.12) that

$$
\begin{array}{r}
\int_{\Pi_{T} \times \Pi_{T}}\left\{\left\langle\nu_{t, x}(\lambda),|\lambda-v|\right\rangle\left(\xi_{t}+\xi_{s}\right)+\right. \\
\left\langle\nu_{t, x}(\lambda), \operatorname{sign}(\lambda-v)(\varphi(\lambda)-\varphi(v))\right\rangle\left(\xi_{x}+\xi_{y}\right)+ \\
\left.|g(u)-g(v)|(\partial / \partial x+\partial / \partial y)^{2} \xi\right\} d t d x d s d y \geq 0 .
\end{array}
$$

We choose now a function $\rho(s) \in C_{0}^{\infty}(\mathbb{R})$ such that $\operatorname{supp} \rho(s) \subset[0,1], \rho(s) \geq 0$, $\int_{-\infty}^{+\infty} \rho(s) d s=1$ and set for $\nu \in \mathbb{N} \delta_{\nu}(s)=\nu \rho(\nu s)$. It is clear that the sequence $\delta_{\nu}(s)$ converges as $\nu \rightarrow \infty$ to the Dirac $\delta$-measure in $\mathcal{D}^{\prime}(\mathbb{R})$. Let $f(t, x) \in C_{0}^{\infty}\left(\Pi_{T}\right)$, $\xi=\xi_{\nu}(t, x ; s, y)=f(t, x) \delta_{\nu}(s-t) \delta_{\nu}(y-x)$. Obviously, $\xi \in C_{0}^{\infty}\left(\Pi_{T} \times \Pi_{T}\right)$ for sufficiently large $\nu, \xi \geq 0$, and

$$
\begin{array}{r}
\xi_{t}+\xi_{s}=f_{t}(t, x) \delta_{\nu}(s-t) \delta_{\nu}(y-x), \quad \xi_{x}+\xi_{y}=f_{x}(t, x) \delta_{\nu}(s-t) \delta_{\nu}(y-x) \\
(\partial / \partial x+\partial / \partial y)^{2} \xi=f_{x x}(t, x) \delta_{\nu}(s-t) \delta_{\nu}(y-x)
\end{array}
$$

Therefore, it follows from (4.13) that

$$
\begin{array}{r}
\int_{\Pi_{T} \times \Pi_{T}}\left\{\left\langle\nu_{t, x}(\lambda),|\lambda-v|\right\rangle f_{t}+\left\langle\nu_{t, x}(\lambda), \operatorname{sign}(\lambda-v)(\varphi(\lambda)-\varphi(v))\right\rangle f_{x}+\right. \\
\left.|g(u)-g(v)| f_{x x}\right\} \delta_{\nu}(s-t) \delta_{\nu}(y-x) d t d x d s d y \geq 0 .
\end{array}
$$

We denote

$$
\begin{array}{r}
R(t, x ; s, y)=\left\langle\nu_{t, x}(\lambda),|\lambda-v(s, y)|\right\rangle f_{t}(t, x)+ \\
\left\langle\nu_{t, x}(\lambda), \operatorname{sign}(\lambda-v(s, y))(\varphi(\lambda)-\varphi(v(s, y)))\right\rangle f_{x}(t, x)+|g(u(t, x))-g(v(s, y))| f_{x x}(t, x) .
\end{array}
$$

Then (4.14) can be written in the form

$$
\int_{\Pi_{T} \times \Pi_{T}} R(t, x ; s, y) \delta_{\nu}(s-t) \delta_{\nu}(y-x) d t d x d s d y \geq 0 .
$$

In view of the obvious estimate:

$$
\begin{array}{r}
|R(t, x ; s, y)-R(t, x ; t, x)| \leq|v(s, y)-v(t, x)|\left|f_{t}(t, x)\right|+ \\
2 \omega(|v(s, y)-v(t, x)|)\left|f_{x}(t, x)\right|+|g(v(s, y))-g(v(t, x))|\left|f_{x x}(t, x)\right|,
\end{array}
$$


where

$$
\omega(\sigma)=\max \{|\varphi(u)-\varphi(v)||u, v \in[-M, M],| u-v \mid \leq \sigma\}
$$

being the continuity modulus of $\varphi(u)$ on the segment $[-M, M]$, we find that for $\nu>(T-t)^{-1}$

$$
\begin{array}{r}
\left|\int_{\Pi_{T}} R(t, x ; s, y) \delta_{\nu}(s-t) \delta_{\nu}(y-x) d s d y-R(t, x ; t, x)\right|= \\
\left|\int_{\Pi_{T}}(R(t, x ; s, y)-R(t, x ; t, x)) \delta_{\nu}(s-t) \delta_{\nu}(y-x) d s d y\right| \leq \\
\int_{\Pi_{T}}|R(t, x ; s, y)-R(t, x ; t, x)| \delta_{\nu}(s-t) \delta_{\nu}(y-x) d s d y \leq \\
\left|f_{t}(t, x)\right| \int_{\Pi_{T}}|v(s, y)-v(t, x)| \delta_{\nu}(s-t) \delta_{\nu}(y-x) d s d y+ \\
2\left|f_{x}(t, x)\right| \int_{\Pi_{T}} \omega(|v(s, y)-v(t, x)|) \delta_{\nu}(s-t) \delta_{\nu}(y-x) d s d y+ \\
\left|f_{x x}(t, x)\right| \int_{\Pi_{T}}|g(v(s, y))-g(v(t, x))| \delta_{\nu}(s-t) \delta_{\nu}(y-x) d s d y \underset{\nu \rightarrow \infty}{\rightarrow} 0
\end{array}
$$

for all Lebesgue points $(t, x)$ of the function $v(t, x)$. In view of $(4.15)$ for a.e. $(t, x) \in$ $\Pi_{T}$

$$
\int_{\Pi_{T}} R(t, x ; s, y) \delta_{\nu}(s-t) \delta_{\nu}(y-x) d s d y \underset{\nu \rightarrow \infty}{\rightarrow} R(t, x ; t, x) .
$$

Integrating this limit relation with respect to $(t, x) \in \Pi_{T}$ with the help of Lebesgue theorem on dominated convergence, we find that

$$
\int_{\Pi_{T} \times \Pi_{T}} R(t, x ; s, y) \delta_{\nu}(s-t) \delta_{\nu}(y-x) d t d x d s d y \underset{\nu \rightarrow \infty}{\rightarrow} \int_{\Pi_{T}} R(t, x ; t, x) d t d x .
$$

From this relation and (4.14) it follows that $\int_{\Pi_{T}} R(t, x ; t, x) d t d x \geq 0$, that is,

$$
\begin{array}{r}
\int_{\Pi_{T}}\left\{\left\langle\nu_{t, x}(\lambda),|\lambda-v|\right\rangle f_{t}+\left\langle\nu_{t, x}(\lambda), \operatorname{sign}(\lambda-v)(\varphi(\lambda)-\varphi(v))\right\rangle f_{x}+\right. \\
\left.|g(u)-g(v)| f_{x x}\right\} d t d x \geq 0
\end{array}
$$

where $v=v(t, x)$. Since $f=f(t, x) \in C_{0}^{\infty}$ is arbitrary non-negative test function then (4.16) is equivalent to (4.4). The proof is complete. $\square$

Since $u, v$ are weak solutions of (1.1) then

$$
(u-v)_{t}+\left(\varphi(u)-\varphi(v)+g(v)_{x}-g(u)_{x}\right)_{x}=0 \text { in } \mathcal{D}^{\prime}\left(\Pi_{T}\right) .
$$

Therefore, there exists a function $P(t, x) \in W_{2, l o c}^{1}\left(\Pi_{T}\right)$ (a potential) such that

$$
P_{x}=u-v, \quad P_{t}=\varphi(v)-\varphi(u)+g(u)_{x}-g(v)_{x} \text { in } \mathcal{D}^{\prime}\left(\Pi_{T}\right) .
$$

This function is Lipschitz continuous with respect to $x$ because $\left|Q_{x}\right|=|u-v| \leq$ $2 M$ and has a strong trace $P(0, x) \in C(\mathbb{R})$. Subtracting a constant from $P$ if necessary, 
we can assume that $P(0,0)=0$. Let us demonstrate that $P(0, x) \equiv 0$. By the construction, $P_{x}(t, \cdot)=(u-v)(t, \cdot) \rightarrow 0$ weakly-* in $L^{\infty}(\mathbb{R})$ as $t \rightarrow 0$ running over some set $E \subset(0,+\infty)$ of full Lebesgue measure. On the other hand, evidently $P_{x}(t, \cdot) \rightarrow P_{x}(0, x)$ in $\mathcal{D}^{\prime}(\mathbb{R})$, and we conclude that $P_{x}(0, x)=0$ in $\mathcal{D}^{\prime}(\mathbb{R})$. Since $P(0, x)$ is continuous, the latter means that $P(0, x) \equiv P(0,0)=0$, as was announced.

LEMMA 4.3. For a.e. $(t, x) \in \Pi_{T}$

$$
\left\langle\nu_{t, x}(\lambda),|\lambda-v|\right\rangle P_{t}+\left\langle\nu_{t, x}(\lambda), \operatorname{sign}(\lambda-v)(\varphi(\lambda)-\varphi(v))\right\rangle P_{x}-|g(u)-g(v)|{ }_{x} P_{x}=0
$$

where $u=u(t, x), v=v(t, x), P=P(t, x)$.

Proof. First, notice that $|g(u)-g(v)|_{x}=\operatorname{sign}(u-v)(g(u)-g(v))_{x}$. Indeed, by the chain rule $|g(u)-g(v)|_{x}=\operatorname{sign}(g(u)-g(v))(g(u)-g(v))_{x}$. But $\operatorname{sign}(g(u)-g(v))=$ $\operatorname{sign}(u-v)$ whenever at least one the values $g(u)$ or $g(v)$ does not belong to the set $E$ of discontinuity points of the function $g_{0}^{-1}$. On the other hand, $g(u)_{x}=g(v)_{x}=0$ for a.e. $(t, x)$ such that $g(u), g(v) \in E$ and $\operatorname{sign}(g(u)-g(v))(g(u)-g(v))_{x}=\operatorname{sign}(u-$ $v)(g(u)-g(v))_{x}=0$.

Using the relation $|g(u)-g(v)|_{x}=\operatorname{sign}(u-v)(g(u)-g(v))_{x}$, we find that for a.e. $(t, x) \in \Pi_{T}$

$$
\begin{array}{r}
\left\langle\nu_{t, x}(\lambda),|\lambda-v|\right\rangle P_{t}+\left\langle\nu_{t, x}(\lambda), \operatorname{sign}(\lambda-v)(\varphi(\lambda)-\varphi(v))\right\rangle P_{x}- \\
|g(u)-g(v)|_{x} P_{x}=\left\langle\nu_{t, x}(\lambda), \operatorname{sign}(\lambda-v)(\varphi(\lambda)-\varphi(v))\right\rangle(u-v)- \\
\left\langle\nu_{t, x}(\lambda),|\lambda-v|\right\rangle\left(\varphi(u)-\varphi(v)-(g(u)-g(v))_{x}\right)-|g(u)-g(v)|_{x}(u-v)= \\
\left\langle\nu_{t, x}(\lambda), \operatorname{sign}(\lambda-v)(\varphi(\lambda)-\varphi(v))\right\rangle(u-v)- \\
\left\langle\nu_{t, x}(\lambda),|\lambda-v|\right\rangle(\varphi(u)-\varphi(v))+\left(\left\langle\nu_{t, x}(\lambda),|\lambda-v|\right\rangle-|u-v|\right)(g(u)-g(v))_{x} .
\end{array}
$$

Let cosupp $\nu_{t, x}=[a(t, x), b(t, x)]$. There are two possible cases: $v(t, x) \notin(a(t, x), b(t, x))$ and $v(t, x) \in(a(t, x), b(t, x))$. In the first case we have

$\left\langle\nu_{t, x}(\lambda),|\lambda-v|\right\rangle=|u-v|,\left\langle\nu_{t, x}(\lambda), \operatorname{sign}(\lambda-v)(\varphi(\lambda)-\varphi(v))\right\rangle=\operatorname{sign}(u-v)(\varphi(u)-\varphi(v))$

(we also use that by Theorem $3.5 \varphi(\lambda)$ is affine on $[a(t, x), b(t, x)]$ ), and (4.17) follows from (4.18).

In the second case $v(t, x) \in(a(t, x), b(t, x))$ and, in particular, $a(t, x)<b(t, x)$. By Theorem 3.5 for a.e. such $(t, x) \quad \varphi(\lambda)=\alpha \lambda+\beta, g(\lambda) \equiv g(u)$ on $[a(t, x), b(t, x)]$, where $\alpha, \beta \in \mathbb{R}$ are constants (depending on $t, x)$. Therefore,

$$
\begin{array}{r}
\left\langle\nu_{t, x}(\lambda), \operatorname{sign}(\lambda-v)(\varphi(\lambda)-\varphi(v))\right\rangle=\alpha\left\langle\nu_{t, x}(\lambda),|\lambda-v|\right\rangle, \\
\varphi(u)-\varphi(v)=\alpha(u-v),
\end{array}
$$

Besides, we see that $g(u)=g(v) \in E$, where $E$ is a set of discontinuity points of the function $g_{0}^{-1}$. Therefore, $g(u)_{x}=g(v)_{x}=0$ for a.e. such $(t, x)$. By (4.18) we see that (4.17) is again satisfied. The proof is complete.

Now we are ready to prove our main result.

THEOREM 4.4. The equality $u=v$ holds a.e. on $\Pi_{T}$. In particular, $u$ is an entropy solution of (1.1) and the trace $u_{0}(x)$ is strong: $\operatorname{ess} \lim u(t, \cdot)=u_{0}$ in $L_{\text {loc }}^{1}(\mathbb{R})$.

Proof. Let $Q(t, x)=q(P(t, x))$, where $q(u)=u^{2} /\left(1+u^{2}\right)$. Then $Q(t, x) \in$ $W_{2, l_{o c}}^{1}\left(\Pi_{T}\right), 0 \leq Q(t, x)<1$, and $Q_{t}=q^{\prime}(P) P_{t}, Q_{x}=q^{\prime}(P) P_{x}$ in $\mathcal{D}^{\prime}\left(\Pi_{T}\right)$. By Lemma 4.3

$$
\left\langle\nu_{t, x}(\lambda),|\lambda-v|\right\rangle Q_{t}+\left\langle\nu_{t, x}(\lambda), \operatorname{sign}(\lambda-v)(\varphi(\lambda)-\varphi(v))\right\rangle Q_{x}-|g(u)-g(v)|_{x} Q_{x}=0
$$


a.e. on $\Pi_{T}$. Then, as follows from Proposition 4.2 and (4.19),

$$
\begin{array}{r}
\frac{\partial}{\partial t}\left(\left\langle\nu_{t, x}(\lambda),|\lambda-v|\right\rangle Q+\varepsilon\right)+\frac{\partial}{\partial x}\left(\left\langle\nu_{t, x}(\lambda), \operatorname{sign}(\lambda-v)(\varphi(\lambda)-\varphi(v))\right\rangle Q\right) \\
-\frac{\partial}{\partial x}\left(|g(u)-g(v)|_{x} Q\right) \leq 0
\end{array}
$$

in $\mathcal{D}^{\prime}\left(\Pi_{T}\right)$ for all $\varepsilon>0$. Let

$$
\begin{array}{r}
\omega_{\varphi}(\sigma)=\max \{|\varphi(u)-\varphi(v)||u, v \in[-M, M],| u-v \mid \leq \sigma\}, \\
\omega_{g}(\sigma)=\max \{|g(u)-g(v)||u, v \in[-M, M],| u-v \mid \leq \sigma\}
\end{array}
$$

be continuity modules of the functions $\varphi(u), g(u)$ on the segment $[-M, M]$, and $\omega(\sigma)=\max \left(\omega_{\varphi}(\sigma), \omega_{g}(\sigma)\right)$. Then $\omega(\sigma)$ is a nondecreasing subadditive function on $[0,+\infty)$ such that $0=\omega(0)=\lim _{\sigma \rightarrow 0+} \omega(\sigma)$, and

$$
|\varphi(u)-\varphi(v)| \leq \omega(|u-v|), \quad|g(u)-g(v)| \leq \omega(|u-v|)
$$

for all $u, v \in[-M, M]$. Observe that for each positive $\varepsilon$

$$
\frac{\omega(\sigma)}{\sigma+\varepsilon} \leq \frac{\omega(\varepsilon)}{\varepsilon} \quad \forall \sigma \geq 0 .
$$

Indeed, we can choose $k \in \mathbb{N}$ such that $\sigma \in[(k-1) \varepsilon, k \varepsilon)$. Then, since $\omega(\sigma)$ is nondecreasing and subadditive, $\omega(\sigma) \leq \omega(k \varepsilon) \leq k \omega(\varepsilon)$ while $\sigma+\varepsilon \geq k \varepsilon$, and (4.21) follows.

Using (4.21), we derive the estimate

$$
\begin{array}{r}
\left|\left\langle\nu_{t, x}(\lambda), \operatorname{sign}(\lambda-v)(\varphi(\lambda)-\varphi(v))\right\rangle Q\right| \leq \\
\left\langle\nu_{t, x}(\lambda),|\varphi(\lambda)-\varphi(v)|\right\rangle Q \leq\left\langle\nu_{t, x}(\lambda), \omega(|\lambda-v|)\right\rangle Q \leq \\
\frac{\omega(\varepsilon)}{\varepsilon}\left(\left\langle\nu_{t, x}(\lambda),|\lambda-v|\right\rangle Q+\varepsilon Q\right) \leq \frac{\omega(\varepsilon)}{\varepsilon}\left(\left\langle\nu_{t, x}(\lambda),|\lambda-v|\right\rangle Q+\varepsilon\right),
\end{array}
$$

where we take into account that $Q<1$. Analogously, we find

$$
\begin{array}{r}
|g(u)-g(v)| Q=\left|\left\langle\nu_{t, x}(\lambda), g(\lambda)-g(v)\right\rangle Q\right| \leq \\
\left\langle\nu_{t, x}(\lambda),|g(\lambda)-g(v)|\right\rangle Q \leq \frac{\omega(\varepsilon)}{\varepsilon}\left(\left\langle\nu_{t, x}(\lambda),|\lambda-v|\right\rangle Q+\varepsilon\right) .
\end{array}
$$

Notice that $Q_{x}=2 P /\left(1+P^{2}\right)^{2} P_{x}=2 P /\left(1+P^{2}\right)^{2}(u-v)$. Therefore,

$$
\left|Q_{x}\right| \leq 2 \sqrt{Q}|u-v| \leq(4 M+2) \varepsilon^{-1 / 2}(Q|u-v|+\varepsilon) .
$$

Indeed, taking into account that $|u-v| \leq 2 M$, we find

$$
\frac{2 \sqrt{Q}|u-v|}{Q|u-v|+\varepsilon} \leq 2 \sqrt{Q}|u-v| / \varepsilon \leq 4 M \varepsilon^{-1 / 2}
$$

if $Q \leq \varepsilon$ while

$$
\frac{2 \sqrt{Q}|u-v|}{Q|u-v|+\varepsilon} \leq 2 / \sqrt{Q} \leq 2 \varepsilon^{-1 / 2}
$$


if $Q \geq \varepsilon$. In the both cases (4.24) is satisfied. Since

$$
|u-v|=\left|\left\langle\nu_{t, x}(\lambda), \lambda-v\right\rangle\right| \leq\left\langle\nu_{t, x}(\lambda),|\lambda-v|\right\rangle,
$$

it follows from (4.24) that

$$
\left|Q_{x}\right| \leq(4 M+2) \varepsilon^{-1 / 2}\left(\left\langle\nu_{t, x}(\lambda),|\lambda-v|\right\rangle Q+\varepsilon\right) .
$$

Now, let

$$
h(s)=\min \left(s^{+}, e^{1-s}\right)=\left\{\begin{array}{lr}
0, & s \leq 0, \\
s, & s \in(0,1), \\
e^{1-s}, & s \geq 1 .
\end{array}\right.
$$

This function is continuous and nonnegative, it increases for $s<1$ and decreases for $s>1$. We set $\rho(r)=\int_{r}^{+\infty} h(s) d s$. Then $\rho(r) \in C^{1}(\mathbb{R}), \rho(r)>0, \rho^{\prime}(r)=-h(r) \leq 0$, and $\rho(r)=$ const $=\int h(s) d s=3 / 2$ for $r \leq 0$. Also notice that the generalized derivative $\rho^{\prime \prime}(r) \in L^{\infty}(\mathbb{R}) \cap L^{1}(\mathbb{R})$ and $\rho^{\prime \prime}(r) \leq 0$ if $r<1, \rho^{\prime \prime}(r)=e^{1-r}>0$ if $r>1$. In particular,

$$
\rho^{\prime \prime}(r) \leq\left|\rho^{\prime}(r)\right|=-\rho^{\prime}(r) \forall r \in \mathbb{R} .
$$

We choose a nonnegative function $\alpha(t) \in C_{0}^{\infty}((0, T))$ and set $f(t, x)=\alpha(t) \rho(|x|+$ $N(t-T))$, where

$$
N=N(\varepsilon)=2 \omega(\varepsilon) / \varepsilon+C \varepsilon^{-1 / 2} .
$$

with $C=(8 M+4) \max _{|u| \leq M}|g(u)|$. Applying (4.20) to the test function $f$, we arrive at the relation

$$
\begin{array}{r}
\left.\int_{\Pi_{T}}\left\langle\nu_{t, x}(\lambda),|\lambda-v|\right\rangle Q+\varepsilon\right) \rho(|x|+N(t-T)) \alpha^{\prime}(t) d t d x+ \\
\int_{\Pi_{T}}\left\{N\left(\left\langle\nu_{t, x}(\lambda),|\lambda-v|\right\rangle Q+\varepsilon\right) \rho^{\prime}(|x|+N(t-T))+\right. \\
\left\langle\nu_{t, x}(\lambda), \operatorname{sign}(\lambda-v)(\varphi(\lambda)-\varphi(v))\right\rangle Q \rho^{\prime}(|x|+N(t-T)) \operatorname{sign} x+ \\
|g(u)-g(v)| Q_{x} \rho^{\prime}(|x|+N(t-T)) \operatorname{sign} x+ \\
\left.|g(u)-g(v)| Q \rho^{\prime \prime}(|x|+N(t-T))\right\} \alpha(t) d t d x \geq 0 .
\end{array}
$$

Notice that $\rho^{\prime}(|x|+N(t-T))=0$ in a vicinity of an interval $\{(t, 0) \mid t \in(0, T)\}$ (where $x=0)$, which implies the equality $(\rho(|x|+N(t-T)))_{x x}=\rho^{\prime \prime}(|x|+N(t-T))$ in $\mathcal{D}^{\prime}\left(\Pi_{T}\right)$. From (4.22),(4.23),(4.25),(4.26), and the trivial estimate $|g(u)-g(v)| \leq 2 \max _{|u| \leq M}|g(u)|$ a.e. on $\Pi_{T}$, we deduce that

$$
\begin{array}{r}
\int_{\Pi_{T}}\left\{N\left(\left\langle\nu_{t, x}(\lambda),|\lambda-v|\right\rangle Q+\varepsilon\right) \rho^{\prime}(|x|+N(t-T))+\right. \\
\left\langle\nu_{t, x}(\lambda), \operatorname{sign}(\lambda-v)(\varphi(\lambda)-\varphi(v))\right\rangle Q \rho^{\prime}(|x|+N(t-T)) \operatorname{sign} x+ \\
|g(u)-g(v)| Q_{x} \rho^{\prime}(|x|+N(t-T)) \operatorname{sign} x+ \\
\left.|g(u)-g(v)| Q \rho^{\prime \prime}(|x|+N(t-T))\right\} \alpha(t) d t d x \leq \\
\int_{\Pi_{T}}\left(\left\langle\nu_{t, x}(\lambda),|\lambda-v|\right\rangle Q+\varepsilon\right)(N-2 \omega(\varepsilon) / \varepsilon- \\
\left.(8 M+4) \max _{|u| \leq M}|g(u)| \varepsilon^{-1 / 2}\right) \rho^{\prime}(|x|+N(t-T)) \alpha(t) d t d x=0
\end{array}
$$


and by (4.27) we claim that

$$
\int_{\Pi_{T}}\left(\left\langle\nu_{t, x}(\lambda),|\lambda-v|\right\rangle Q+\varepsilon\right) \rho(|x|+N(t-T)) \alpha^{\prime}(t) d t d x \geq 0,
$$

that is, $\int_{0}^{T} I(t) \alpha^{\prime}(t) d t \geq 0 \forall \alpha(t) \in C_{0}^{\infty}((0, T)), \alpha(t) \geq 0$, where

$$
I(t)=\int_{\mathbb{R}}\left(\left\langle\nu_{t, x}(\lambda),|\lambda-v|\right\rangle Q+\varepsilon\right) \rho(|x|+N(t-T)) d x .
$$

This means that $I^{\prime}(t) \leq 0$ in $\mathcal{D}^{\prime}((0, T))$. Therefore, for a.e. $t \in(0, T)$

$$
\begin{array}{r}
I(t) \leq \underset{t \rightarrow 0}{\operatorname{ess} \lim } I(t) \leq \underset{t \rightarrow 0}{\operatorname{ess} \lim } \int_{\mathbb{R}}(2 M Q(t, x)+\varepsilon) \rho(|x|+N(t-T)) d x= \\
\varepsilon \int_{\mathbb{R}} \rho(|x|-N T) d x .
\end{array}
$$

We use here the fact that the strong trace of $Q$ at the line $t=0$ equals $q(P(0, x))=0$, i.e., $\underset{t \rightarrow 0}{\operatorname{ess} \lim } Q(t, \cdot)=0$ in $L_{l o c}^{1}(\mathbb{R})$. Since $0 \leq Q<1$ and $\rho(|x|+N(t-T)) \leq \rho(|x|-$ $N T)) \in L^{1}(\mathbb{R})$ we claim that $\underset{t \rightarrow 0}{\operatorname{ess} \lim } \int_{\mathbb{R}} Q(t, x) \rho(|x|+N(t-T)) d x=0$ and the last equality in (4.28) follows. Now observe that $\rho(r) \leq 3 / 2$ and $\rho(r)=e^{1-r}$ for $r>1$. Hence,

$$
\int_{\mathbb{R}} \rho(|x|-N T) d x \leq \frac{3}{2} \int_{-N T-1}^{N T+1} d x+2 \int_{1}^{+\infty} e^{1-r} d r=3 N T+5 .
$$

In view of (4.28) for every $t \in(0, T)$, being a Lebesgue point of the function

$$
\begin{array}{r}
\int_{\mathbb{R}}\left\langle\nu_{t, x}(\lambda),|\lambda-v|\right\rangle Q(t, x) \rho(|x|+N(t-T)) d x, \text { we have } \\
\int_{\mathbb{R}}\left\langle\nu_{t, x}(\lambda),|\lambda-v|\right\rangle Q(t, x) \rho(|x|+N(t-T)) d x \leq I(t) \leq \\
3 T N(\varepsilon) \varepsilon+5 \varepsilon=3 T(2 \omega(\varepsilon)+C \sqrt{\varepsilon})+5 \varepsilon \underset{\varepsilon \rightarrow 0}{\rightarrow} 0 .
\end{array}
$$

This readily implies that

$$
\int_{\Pi_{T}}\left\langle\nu_{t, x}(\lambda),|\lambda-v|\right\rangle Q(t, x) \rho(|x|+N(t-T)) d t d x=0
$$

and since $\rho(|x|+N(t-T))>0$ we see that

$$
\left\langle\nu_{t, x}(\lambda),|\lambda-v(t, x)|\right\rangle Q(t, x)=0 \quad \text { a.e. on } \Pi_{T} .
$$

By (4.29) we find that $\left\langle\nu_{t, x}(\lambda),|\lambda-v(t, x)|\right\rangle=0$ a.e. on the set $\{P(t, x) \neq 0\}$. This implies that $v(t, x)=u(t, x)=\left\langle\nu_{t, x}(\lambda), \lambda\right\rangle$ a.e. on this set. On the other hand, a.e. on the set $\{P(t, x)=0\}$, we have $u(t, x)-v(t, x)=P_{x}(t, x)=0$. Thus, $u(t, x)=v(t, x)$ a.e. on $\Pi_{T}$. In particular, $u$ is an e.s. of the Cauchy problem for (1.1) with initial data $u_{0}(x)$. The proof is complete.

Remark 2. As was shown in [13] (also see [14]), the existence of strong traces remains valid for entropy solutions of conservation laws even in the multidimensional case. 
Let us consider now the more general equation

$$
\alpha(u)_{t}+\varphi(u)_{x}-g(u)_{x x}=0
$$

where $\alpha(u)$ is a continuous strictly increasing function. The notion of entropy solution $u=u(t, x)$ of $(4.30)$ is defined by a relation similar to (1.4): for all $k \in \mathbb{R}$

$$
|\alpha(u)-\alpha(k)|_{t}+[\operatorname{sign}(u-k)(\varphi(u)-\varphi(k))]_{x}-|g(u)-g(k)|_{x x} \leq 0 \text { in } \mathcal{D}^{\prime}\left(\Pi_{T}\right) .
$$

As was shown in [15], the statement of Theorem 4.4 is no longer valid for equation (4.30) if $\alpha(u)$ is not linear. Moreover, it may occur that a weak limit of a sequence of e.s. to (4.30) is not even a weak solution of this equation. But, noticing that $u=u(t, x)$ is an entropy solution of (4.30) if and only if $v=g(u)$ is an entropy solution to the standard equation

$$
v_{t}+\varphi\left(\alpha^{-1}(v)\right)_{x}-g\left(\alpha^{-1}(v)\right)_{x x}=0,
$$

we can revise the assertion of Theorem 4.4 for equation (4.30) (in the case of conservation laws this was done in [15, Theorem 3.1]). Thus, we consider approximate equations

$$
\alpha_{n}(u)_{t}+\varphi_{n}(u)_{x}-g_{n}(u)_{x x}=0, n \in \mathbb{N},
$$

where the sequences $\alpha_{n}(u), \varphi_{n}(u), g_{n}(u) \in C(\mathbb{R}), g_{n}(u)$ are increasing and $\alpha_{n}(u)$ are strictly increasing functions. Assume that these sequences converges as $n \rightarrow \infty$ to the functions $\alpha(u), \varphi(u), g(u)$, respectively, in the space $C(\mathbb{R})$ (i.e., uniformly on any segment).

THEOREM 4.5. Let $u_{n}=u_{n}(t, x), n \in \mathbb{N}$, be a bounded sequence of entropy solutions to (4.31) such that the sequence $v_{n}=\alpha_{n}\left(u_{n}\right)$ weakly-* converges in $L^{\infty}\left(\Pi_{T}\right)$ to a function $v=v(t, x)$. Then $v=\alpha(u)$, where $u=u(t, x)$ is an entropy solution of (4.30). Moreover, $u$ satisfies the initial condition with some function $u_{0}(x)$ in the sense of relation (1.7).

Proof. Let $M=\sup \left\|u_{n}\right\|_{\infty}, s_{n}(v)=s_{\alpha_{n}(-M), \alpha_{n}(M)}(v), \tilde{\varphi}_{n}(v)=\varphi_{n}\left(\left(\alpha_{n}\right)^{-1}\left(s_{n}(v)\right)\right)$, $\tilde{g}_{n}(v)=g_{n}\left(\left(\alpha_{n}\right)^{-1}\left(s_{n}(v)\right)\right)$. One can easily verify that $\tilde{\varphi}_{n}(v) \rightarrow \tilde{\varphi}(v) \doteq \varphi\left(\alpha^{-1}(s(v))\right.$, $\tilde{g}_{n}(v) \rightarrow \tilde{g}(v) \doteq g\left(\alpha^{-1}(s(v))\right.$ as $n \rightarrow \infty$ in $C(\mathbb{R})$, where we denote $s(v)=s_{\alpha(-M), \alpha(M)}(v)$. Notice that $v_{n}=\alpha_{n}\left(u_{n}\right)$ are entropy solutions of the equations

$$
v_{t}+\tilde{\varphi}_{n}(v)_{x}-\tilde{g}_{n}(v)_{x x}=0,
$$

and $v_{n}(t, x) \in\left[\alpha_{n}(-M), \alpha_{n}(M)\right]$ a.e. on $\Pi_{T}$. By Theorem 4.4 we claim that the weak- $*$ limit $v=v(t, x)$ of this sequence is an entropy solution to the Cauchy problem for the equation

$$
v_{t}+\tilde{\varphi}(v)_{x}-\tilde{g}(v)_{x x}=0,
$$

with an initial data $v_{0}(x)$. Evidently, $v(t, x) \in[\alpha(-M), \alpha(M)]$ a.e. on $\Pi_{T}$, and $v_{0}(x) \in[\alpha(-M), \alpha(M)]$ a.e. on $\mathbb{R}$. Therefore, $v=\alpha(u(t, x))$ a.e. on $\Pi_{T}$, where $u=u(t, x) \in L^{\infty}\left(\Pi_{T}\right),\|u\|_{\infty} \leq M$. Then, to conclude the proof, it only remains to observe that $u(t, x)$ is an entropy solution of (4.30), satisfying the initial condition $u(0, \cdot)=u_{0}=\alpha^{-1}\left(v_{0}(x)\right)$.

Remark 3. Certainly, our results are purely one-dimensional. The statement of Theorem 4.4 is not true for multidimensional equations even with only one nonlinear 
flux component. For instance, it was shown in [15] that there exists a sequence $u_{n}(t, x, y)$ of entropy solutions of the conservation law $u_{t}+f(u)_{x}=0, u=u(t, x, y)$, with nonlinear flux $f(u)$ such that $u_{n} \rightarrow u=u(t, x, y)$ as $n \rightarrow \infty$ but $u$ is not a weak solution of this equation.

\section{REFERENCES}

[1] B. Andreianov And M. Maliki, A note on uniqueness of entropy solutions to degenerate parabolic equations in $\mathbb{R}^{N}$, NoDEA: Nonlin. Diff. Eq. Appl., 17 (2010), no. 1, pp. 109-118.

[2] J. CARrillo, Entropy solutions for nonlinear degenerate problems, Arch. Rational Mech. Anal., 147(1999), pp. 269-361.

[3] G.-Q. Chen AND H. FRID, Divergence-measure fields and hyperbolic conservation laws, Arch. Ration. Mech. Anal., 147 (1999), pp. 89-118.

[4] G.-Q. Chen AND Y.-G. LU, The study on application way of the compensated compactness theory, Chinese Sci. Bull., 34 (1989), pp. 15-19.

[5] C. M. Dafermos, Hyperbolic Conservation Laws in Continuum Physics, 3rd ed., SpringerVerlag, Berlin, 2010.

[6] R. J. DiPerna, Measure-valued solutions to conservation laws, Arch. Rational Mech. Anal., 88 (1985), pp. 223-270.

[7] L. C. Evans And R. F. Gariepy, Measure Theory and Fine Properties of Functions, CRC Press, Boca Raton, FL, 1992.

[8] H. Holden, K. H. Karlsen, D. Mitrovič, E. Yu. Panov, Strong compactness of approximate solutions to degenerate elliptic-hyperbolic equations with discontinuous flux function, Acta Mathematica Scientia 29B (2009), no. 6, pp. 1573-1612.

[9] S. N. KRuzhkov, First order quasilinear equations in several independent variables, Mat. Sb. (N.S.), 81 (1970), pp. 228-255 (English transl. in Math. USSR-Sb., 10 (1970), pp. 217-243).

[10] M. MALIKI AND H. TOURÉ, Uniqueness of entropy solutions for nonlinear degenerate parabolic problem, J. Evol. Equ., 3 (2003), no.4, pp. 603-622.

[11] F. Murat, Compacité par compensation, Ann. Scuola Norm. Sup. Pisa Cl. Sci. (4), 5 (1978), pp. $489-507$.

[12] F. Murat, L'injection du cône positif de $H^{-1}$ dans $W^{-1, q}$ est compacte pour tout $q<2$, J. Math. Pures Appl. (9), 60 (1981), no. 3, pp. 309-322.

[13] E. Yu. PANOV, Existence of strong traces for generalized solutions of multidimensional scalar conservation laws, J. Hyperbolic Differ. Equ., 2 (2005), pp. 885-908.

[14] E. YU. PANOV, Existence of strong traces for quasi-solutions of multidimensional scalar conservation laws, J. Hyperbolic Differ. Equ., 4 (2007), pp. 729-770.

[15] E. Yu. PANov, On weak completeness of the set of entropy solutions to a scalar conservation law, SIAM J. Math. Anal., 41 (2009), no. 1, pp. 26-36.

[16] E. YU. PANOV, On the strong pre-compactness property for entropy solutions of a degenerate elliptic equation with discontinuous flux, J. Differential Equations 247 (2009), no. 10, pp. 2821-2870.

[17] E. Yu. Panov, Ultra-parabolic H-measures and compensated compactness, Annales de l'Institut Henri Poincare (C) Analyse Non Lineaire, 28 (2011), no. 1, pp. 47-62.

[18] D. Serre, Systems of Conservation Laws. Vol. 2. Geometric Structures, Oscillations, and Initial-Boundary Value Problems, Cambridge University Press, Cambridge, UK, 1999.

[19] L. TARTAR, Compensated compactness and applications to partial differential equations, in Nonlinear Analysis and Mechanics: Heriot-Watt Symposium, Vol. 4 (Edinburgh 1979), Res. Notes in Math. 39, Pitman, Boston, 1979, pp. 136-212. 